\title{
On Fermi systems with strong forward scattering in $d$ spatial dimensions
}

\author{
BEHNAM FARID \\ Cavendish Laboratory, Department of Physics, University of Cambridge, \\ Cambridge CB3 OHE, United Kingdom* \\ and Max-Planck-Institut für Festkörperforschung, Heisenbergstraße 1, \\ 70569 Stuttgart, Federal Republic of Germany ${ }^{\dagger}$
}

(October 30, 2018)

\begin{abstract}
We consider a specific generalisation to spatial dimensions $d$ greater than one of a formalism based upon conservation laws and the associated Ward identities, that exactly solves the one-dimensional Luttinger model, and expose its inadequacies for $d>1$. We conclude that findings arrived at through application of this generalised formalism concerning systems of fermions with strong forward scattering, in particular that $d=1$ would be the cross-over dimension from Luttinger- to Fermi-liquid state, are open to question.
\end{abstract}

To appear in: Philosophical Magazine, Part B, Vol. 80, No. 9 (2000)

\section{INTRODUCTION}

The question with regard to the existence of non-Fermi liquid (NFL) metallic states in spatial dimensions $d$ greater than one, specifically in $d=2$, has been subject of intensive investigations in the course of the past several years, following the observation that the normal-state properties of doped cuprate superconducting compounds markedly differ from those of conventional metals which are accurately described within the framework of the Fermi-liquid (FL) theory (Anderson 1988,1989,1990a,b,1997, Varma, Littlewood, Schmitt-Rink, Abrahams and Ruckenstein 1989, 1990, Littlewood and Varma 1991). Since according to a celebrated theorem due to Luttinger (1961), validity of the many-body perturbation theory to all orders would imply that metallic states of fermions in spatial dimensions greater than one would be FLs, 1 failure of the FL picture in the case of the doped cuprates has been considered to signal breakdown of the many-body perturbation theory as applied to these materials. Indeed, in the course of the recent years the question with regard to breakdown of the many-body perturbation theory has been a central aspect in debates concerning the above-mentioned failure of the FL phenomenology in describing the normal-state properties of the doped cuprate compounds (Anderson 1990b, 1993, Engelbrecht and Randeria 1990, Stamp 1993) (see also Engelbrecht and Randeria (1991), Anderson (1991)). Consequently, in this period a number of non-perturbative methods have been put forward and applied to models of interacting fermions with the aim of determining whether or not NFL states are viable in $d>1$. Elsewhere (Farid 1999a,b) we have presented accounts of these developments and therefore refrain from repetition here. For our present considerations it is relevant to mention that the existence of NFL metallic states in realistic microscopic models of interacting fermions in $d>1$ has remained elusive to this date.

In view of the above-indicated apparent discrepancy that prevails between theoretical predictions and experimental observations, we have undertaken to subject a number of aspects that are crucial to establishing feasibility or otherwise of NFL metallic states in $d>1$ to an independent investigation. The present work is the third report of our investigations on this subject. Our first work (Farid 1999a) concerned a critical analysis of the above-indicated

\footnotetext{
${ }^{*}$ Electronic address: bf10000@phy.cam.ac.uk

${ }^{\dagger}$ Electronic address: farid@audrey.mpi-stuttgart.mpg.de

${ }^{1}$ Strictly speaking, this theorem has direct bearing on cases where fermions interact through a short-range two-body potential.
} 
Luttinger (1961) theorem. We have demonstrated (Farid 1999a) that Luttinger's (1961) proof of this theorem involves an implicit assumption which is strictly specific to FL metallic states, implying that the Luttinger (1961) theorem far from excluding the possibility of the existence of NFL metallic states in $d>1$ (for cases where the many-body perturbation theory is valid to all orders), in fact amounts to a statement of consistency of the FL states with the principles of the theory of interacting fermions as expressed in terms of the many-body perturbation theory.

Our second work (Farid 1999b) concerned an in-depth analysis of the non-perturbative bosonisation scheme due to Haldane (1992) for metallic states in $d \geq 1$ as applied to a model of interacting fermions in $d=2$, originally put forward by Houghton and Marston (1993) and subsequently extensively studied by Houghton, Kwon and Marston (1994), Houghton, Kwon, Marston and Shankar (1994) and Kwon, Houghton and Marston (1995). We have demonstrated failure of the Haldane (1992) bosonisation scheme in $d>1$ and established that this scheme invariably predicts the metallic state of the model under consideration to be an unconventional FL, irrespective of the nature (and strength, provided it be non-vanishing) of the fermion-fermion interaction function, that is whether this be shortrange, long-range Coulomb or super-long range. The latter result is in particular at variance with an earlier finding by Bares and Wen (1993), 月 indicating the metallic state of the system for super-long-range interactions to be a NFL. We have traced back the shortcoming of the Haldane (1992) bosonisation scheme in $d>1$ to the neglect of some terms in the commutation relation concerning some current operators; in particular, in the case of metallic systems with a sufficiently small (or vanishing) value for the jump $Z_{\mathbf{k}_{F}}$ in the momentum distribution function at the Fermi momentum, the neglected terms are more relevant than the sole term that is taken into account, namely the quantum anomaly. 3 We have also shown that under all conditions the Fermi energy of the interacting system within the Haldane (1992) bosonisation scheme coincides with that of the non-interacting system. This unambiguously implies that even for weakly-interacting metallic states, for which $Z_{\mathbf{k}_{F}}$ is close to unity, neglect of the indicated terms in the mentioned commutation relation for current operators is not justified.

The exactly solvable one-dimensional Luttinger model (Luttinger 1963, Mattis and Lieb 1965) has been dealt with by three distinct methods. One is based on the technique of bosonisation, of which the Haldane (1992) scheme is a generalisation to higher dimensions. If The second, due to Everts and Schulz (1974), is based on the solution of the equation of motion for the Green function which derives from an exact decoupling of the hierarchy of the Green functions. The third approach, due to Dzyaloshinskii and Larkin (DL) (1974), f exploits the conservation laws and the associated Ward identities. This scheme has been generalised to $d>1$ and reported in a series of articles by Di Castro and Metzner (1991), Metzner and Di Castro (1993), Castellani, Di Castro and Metzner (1994), Castellani and Di Castro (1994) and extensively reviewed by Metzner, Castellani and Di Castro (MCDC) (1998). It is this scheme that we subject to a critical analysis in the present work. In our considerations that follow, we shall almost exclusively refer to the last review article without thereby implying that the cited subject matters would have their origin in this work.

Contrary to case of $d=1$, associated with linear energy dispersions for the non-interacting left- and right-movers (that is the case of the one-dimensional Luttinger (1963) model), in cases of $d>1$ the generalised DL approach is not exact; MCDC (1998) assert, however, that for Fermi systems the generalised approach is asymptotically exact in the limit of strong forward scattering, that is in the limit of zero momentum transfer $\mathbf{q}$ for fermions in a narrow band of width $\lambda$ ( $\Lambda$ in the authors' notation) encompassing the Fermi surface. In this work we demonstrate this not to be the case. We further support this finding by referring to the similarity between the expressions for the single-particle Green function within the framework of the generalised DL (MCDC 1998) scheme and the Haldane (1992) bosonisation approach and the fact that, as we have indicated above, the latter is not adequate for $d>1$ (Farid 1999b). We also establish that the rigorous formalism for systems of interacting fermions, even in the limit of strong forward scattering, is not amenable to treatment by the Wilson-Fisher (1972) (Wilson 1973) technique of

\footnotetext{
${ }^{2}$ It is also at variance with the findings by Houghton, Kwon, Marston and Shankar (1994) and Kwon, Houghton and Marston (1995). We have however shown (Farid 1999b) that this discrepancy is due to use of certain invalid approximations by the latter authors.

${ }^{3}$ For completeness, we mention that by solely taking into account the quantum anomaly, the mentioned current operators form a Kac-Moody algebra (see, e.g., Goddard and Olive 1986).

4 The bosonisation method for calculation of correlation functions is due to Luther (1979) which derives from works by Luther and Peschel (1974), Mattis and Lieb (1965), Tomonaga (1950) and Bloch (1933, 1934). Parallel developments involving similar views as in the indicated works are due to Jordan (1935, 1936a,b), Krönig (1935a,b,c), Skyrme (1958, 1959, 1961a,b) and Coleman (1975). For reviews see Stone (1994) and Kopietz (1997).

${ }^{5}$ For a detailed exposition of this approach see (Bohr 1981).
} 
dimensional continuation.

The organisation of this work is as follows. In $\S$ II we present an outline of the generalised DL formalism (MCDC 1998) which will facilitate our discussions in this paper. Anticipating the results that we present in $\S$ III, here we indicate that the conventional process of dimensional continuation is not applicable to a general formalism concerning systems of interacting fermions, not even in the limit where solely forward scattering processes are taken into account. In $\S$ III we analyse two crucial steps that are involved in the derivation of the generalised DL formalism and explicitly demonstrate their inadequacies in $d>1$; in $\S$ III.A we deal with a relationship between the charge and the current part of a vertex function that has been held to be asymptotically correct in the limit of strong forward-scattering and show that its validity in $d>1$ is strictly limited to the trivial case of non-interacting fermions; in $\S$ III.B we show the invalidity of a second relationship between two irreducible vertex functions, also held to be asymptotically correct in $d>1$ in the limit of strong forward-scattering. In $\S$ IV we explicitly demonstrate how the singular behaviour of the momentum distribution function, in particular that pertaining to the ground-state of the system, at the Fermi momentum, renders the mathematical manipulations that are central to the derivation of the generalised DL method in $d>1$ (MCDC 1998) inapplicable. Here we further show how similar manipulations, combined with a complete disregard of the errors associated with these, in $d=1$ (specifically in dealing with the one-dimensional Luttinger model) give rise to results which coincide with the exact results, thus misleadingly suggesting their general applicability even in $d>1$. We finally present a summary and concluding remarks of this work in $\S \mathrm{V}$. We devote an Appendix which follows the main text to a brief exposition of the Wilson-Fisher (Wilson and Fisher 1972, Wilson 1973) technique of dimensional continuation, which concerns continuation of the number of the spatial dimensions from integer to real, as well as complex, values. From the details in this Appendix it becomes apparent how application of this technique is hampered in the context of the many-body theory of interacting fermions. Here we propose an alternative scheme of dimensional continuation which, although not unique, is not restrictive with regard to the functional dependence of the integrands on the variable of integration.

\section{OUTLINE OF THE GENERALISED DZYALOSHINSKIII-LARKIN SCHEME}

Here we present a brief outline of the generalised DL scheme. For a detailed exposition of this scheme readers are referred to the article by MCDC (1998). In this Section we adopt the notational conventions of the latter work. For completeness, below $p$ and $q$ denote $d+1$-vectors $\left(i p_{0}, \mathbf{p}\right)$ and $\left(i q_{0}, \mathbf{q}\right)$ respectively, with $p_{0}\left(q_{0}\right)$ energy (throughout we assume $\hbar=1$ ) and $\mathbf{p}(\mathbf{q})$ momentum in $d$-dimensional momentum space (here $d$ is integer; we shall be explicit when $d$ is non-integer); $\left(i p_{0}, \mathbf{p}\right)_{\nu}$ represents $i p_{0}$ when $\nu=0$ and $-p_{\nu}$ when $\nu=1,2, \ldots, d ; \int_{k} f(k)$, with $f(k) \equiv F\left(i k_{0} ; \mathbf{k}\right)$, is the short for $(2 \pi)^{-1} \int \mathrm{d} k_{0}(2 \pi)^{-d} \int \mathrm{d}^{d} k F\left(i k_{0} ; \mathbf{k}\right)$. For finite temperatures $T,(2 \pi)^{-1} \int \mathrm{d} k_{0}(\ldots)$ symbolises a Matsubara sum, $(2 \pi)^{-1} \int \mathrm{d} k_{0}(\ldots) \rightarrow T \sum_{k_{0}}(\ldots)$, where the summation runs over the discrete fermion Matsubara energies $2 \pi i\left(k_{0}+1 / 2\right) T$, with $k_{0}=0, \pm 1, \ldots$. Below, unless specifically indicated otherwise, we assume the summation convention $A_{\nu} B^{\nu} \equiv \sum_{\nu=0}^{d} A_{\nu} B^{\nu}$.

For a system whose Hamiltonian $\hat{H}$ is of the form

$$
\hat{H} \equiv \hat{H}_{0}+\hat{H}_{I}:=\sum_{\mathbf{k} \sigma} \varepsilon_{\mathbf{k}}^{0} \hat{a}_{\mathbf{k}, \sigma}^{\dagger} \hat{a}_{\mathbf{k}, \sigma}+\frac{1}{2 V} \sum_{\mathbf{k}, \mathbf{k}^{\prime}, \mathbf{q}} \sum_{\sigma \sigma^{\prime}} g_{\mathbf{k} \mathbf{k}^{\prime}}^{\sigma}(\mathbf{q}) \hat{a}_{\mathbf{k}-\mathbf{q} / 2, \sigma}^{\dagger} \hat{a}_{\mathbf{k}+\mathbf{q} / 2, \sigma} \hat{a}_{\mathbf{k}^{\prime}+\mathbf{q} / 2, \sigma^{\prime}}^{\dagger} \hat{a}_{\mathbf{k}^{\prime}-\mathbf{q} / 2, \sigma^{\prime}}
$$

with $\hat{a}_{\mathbf{k}, \sigma}^{\dagger}, \hat{a}_{\mathbf{k}, \sigma}$ the canonical creation and annihilation operators for fermions with spin $\sigma$, respectively, $\varepsilon_{\mathbf{k}}^{0}$ the energy dispersion of the non-interacting fermions, 16 volume of the system and $g_{\mathbf{k} \mathbf{k}^{\prime}}^{\sigma \sigma^{\prime}}(\mathbf{q})$ the fermion-fermion interaction function, MCDC (1998) attempt to transform the expression for the self-energy $\Sigma_{\sigma}(p)$,

$$
\Sigma_{\sigma}(p)=-\sum_{\sigma^{\prime}} \int_{p^{\prime}} D_{\mathbf{p} \mathbf{p}^{\prime}}^{\sigma \sigma^{\prime}}\left(p-p^{\prime}\right) G_{\sigma}\left(p^{\prime}\right) \Lambda_{\sigma^{\prime} \sigma}^{0}\left(\left[p+p^{\prime}\right] / 2 ; p^{\prime}-p\right)
$$

into a closed form. Here $D_{\mathbf{p} \mathbf{p}^{\prime}}^{\sigma}\left(p-p^{\prime}\right)$ is the screened interaction function (see Eq. (17) below), $G_{\sigma}(k) \equiv G_{\sigma}\left(i k_{0} ; \mathbf{k}\right)$ the (thermal) single-particle Green function,

\footnotetext{
${ }^{6}$ We assume $\varepsilon_{\mathbf{k}}^{0} \equiv\|\mathbf{k}\|^{2} / 2$ (in units where $m_{f}$, the fermion mass, is equal to unity). For some relevant remarks see Footnote 12 further on.
} 


$$
\Lambda_{\sigma^{\prime} \sigma}^{0}(p ; q):=\left\langle\hat{n}_{\sigma^{\prime}}(q) \hat{a}_{p-q / 2, \sigma} \hat{a}_{p+q / 2, \sigma}^{\dagger}\right\rangle_{\mathrm{tr}}^{\mathrm{irr}}
$$

the irreducible density (or charge) vertex, in which 7

$$
\hat{n}_{\sigma}(\mathbf{q}):=\sum_{\mathbf{k}} \hat{a}_{\mathbf{k}-\mathbf{q} / 2, \sigma}^{\dagger} \hat{a}_{\mathbf{k}+\mathbf{q} / 2, \sigma}
$$

In Eq. (3) "tr" indicates that the external Green functions are not taken into account (i.e. they are truncated), which amounts to dividing the expression associated with the (thermal) average $\langle. . .\rangle^{\text {irr }}$, without subscript "tr", by $G_{\sigma}(p+q / 2) G_{\sigma}(p-q / 2)$; the superscript "irr" indicates that in the diagrammatic expansion of the associated correlation function only one-interaction-irreducible diagrams are taken into account, i.e. those which do not fall into two disjoint parts upon removing an interaction line in them. Through reliance upon the generalised closed-loop theorem (CLT) (Kopietz, Hermisson and Schönhammer 1995), \& MCDC (1998) relate $\Lambda_{\sigma^{\prime} \sigma}^{\nu}(p ; q)$ with the irreducible vertex part associated with the non-interacting density-current $\hat{j}_{0}^{\nu}(q)$, that is

$$
\Lambda_{\sigma}^{\nu}(p ; q):=\left\langle\hat{j}_{0}^{\nu}(q) \hat{a}_{p-q / 2, \sigma} \hat{a}_{p+q / 2, \sigma}^{\dagger}\right\rangle_{\mathrm{tr}}^{\mathrm{irr}}
$$

as follows:

$$
\Lambda_{\sigma^{\prime} \sigma}^{0}(p ; q)=\delta_{\sigma^{\prime}, \sigma} \Lambda_{\sigma}^{0}(p ; q), \quad \boldsymbol{\Lambda}_{\sigma^{\prime} \sigma}(p ; q)=\delta_{\sigma^{\prime}, \sigma} \Lambda_{\sigma}^{0}(p ; q) \mathbf{v}_{\mathbf{p}}^{0}
$$

Here the $d$-dimensional vector $\boldsymbol{\Lambda}_{\sigma^{\prime} \sigma}(p ; q)$ corresponds to the $\nu=1,2, \ldots, d$ components of $\Lambda_{\sigma^{\prime} \sigma}^{\nu}(p ; q)$. The expressions in Eq. (6) can be written as

$$
\Lambda_{\sigma^{\prime} \sigma}^{0}(p ; q)=\delta_{\sigma^{\prime}, \sigma} \frac{\left(i q_{0}, \mathbf{q}\right)_{\nu} \Lambda_{\sigma}^{\nu}(p ; q)}{i q_{0}-\mathbf{q} \cdot \mathbf{v}_{\mathbf{p}}^{0}}
$$

The density-current vertex function $\Lambda_{\sigma}^{\nu}(p ; q)$ is related to the vertex part

$$
\Gamma_{\sigma}^{\nu}(p ; q):=\left\langle\hat{j}^{\nu}(q) \hat{a}_{p-q / 2, \sigma} \hat{a}_{p+q / 2, \sigma}^{\dagger}\right\rangle_{\mathrm{tr}}
$$

and the irreducible vertex part corresponding to the interacting density-current $\hat{j}^{\nu}(q)$, that is

$$
\Lambda_{\sigma}^{\prime}{ }^{\nu}(p ; q):=\left\langle\hat{j}^{\nu}(q) \hat{a}_{p-q / 2, \sigma} \hat{a}_{p+q / 2, \sigma}^{\dagger}\right\rangle_{\mathrm{tr}}^{\mathrm{irr}},
$$

through the Dyson equation

$$
\Gamma_{\sigma}^{\nu}(p ; q)=\Lambda_{\sigma}^{\prime}{ }^{\nu}(p ; q)+\frac{1}{V} \sum_{\mathbf{k}^{\prime} \sigma^{\prime}} \sum_{\mathbf{k}^{\prime \prime} \sigma^{\prime \prime}} J^{\nu, \mathbf{k}^{\prime} \sigma^{\prime}}(q) g_{\mathbf{k}^{\prime} \mathbf{k}^{\prime \prime}}^{\sigma^{\prime \prime}}(q) \Lambda_{\sigma}^{\mathbf{k}^{\prime \prime} \sigma^{\prime \prime}}(p ; q),
$$

where

$$
\begin{gathered}
J^{\nu, \mathbf{k} \sigma}(q):=-\int \frac{\mathrm{d} k_{0}}{2 \pi}\left\langle\hat{j}^{\nu}(q) \hat{a}_{k+q / 2, \sigma}^{\dagger} \hat{a}_{k-q / 2, \sigma}\right\rangle \equiv \int \frac{\mathrm{d} k_{0}}{2 \pi} \Gamma_{\sigma}^{\nu}(k ; q) G_{\sigma}(k+q / 2) G_{\sigma}(k-q / 2), \\
\Lambda_{\sigma}^{\mathbf{k} \sigma^{\prime}}(p ; q):=\int \frac{\mathrm{d} k_{0}}{2 \pi}\left\langle\hat{a}_{k-q / 2, \sigma^{\prime}}^{\dagger} \hat{a}_{k+q / 2, \sigma^{\prime}} \hat{a}_{p-q / 2, \sigma} \hat{a}_{p+q / 2, \sigma}^{\dagger}\right\rangle_{\mathrm{tr}}^{\mathrm{irr}} .
\end{gathered}
$$

Owing to the exact Ward identity

$$
\left(i q_{0}, \mathbf{q}\right)_{\nu} \Gamma_{\sigma}^{\nu}(k ; q)=G_{\sigma}^{-1}(k+q / 2)-G_{\sigma}^{-1}(k-q / 2),
$$

\footnotetext{
7 Note that $\sum_{\sigma} \hat{n}_{\sigma}(q) \equiv \hat{j}_{0}^{0}(q) \equiv \hat{j}^{0}(q)$, where $\hat{j}_{0}^{0}(q)$ and $\hat{j}^{0}(q)$ stand for the density (or charge) parts of $\hat{j}_{0}^{\nu}(q)$ and $\hat{j}^{\nu}(q)$, respectively (see Eqs. (18) and (43) below).

8 The CLT (or, loop-cancellation theorem) for the one-dimensional Luttinger (1963) model is due to Dzyaloshinskii and Larkin (1974). For an extensive exposition of this theorem see Bohr (1981).
} 
from Eq. 11] it readily follows that

$$
\left(i q_{0}, \mathbf{q}\right)_{\nu} J^{\nu, \mathbf{k} \sigma}(q)=\mathrm{n}_{\sigma}(\mathbf{k}-\mathbf{q} / 2)-\mathrm{n}_{\sigma}(\mathbf{k}+\mathbf{q} / 2)
$$

where

$$
\mathrm{n}_{\sigma}(\mathbf{k} \pm \mathbf{q} / 2):=\left\langle\hat{a}_{\mathbf{k} \pm \mathbf{q} / 2, \sigma}^{\dagger} \hat{a}_{\mathbf{k} \pm \mathbf{q} / 2, \sigma}\right\rangle \equiv \int \frac{\mathrm{d} k_{0}}{2 \pi} G_{\sigma}(k \pm q / 2)
$$

stands for the momentum distribution function corresponding to the spin- $\sigma$ fermions. Through relying upon (see however $\S \S$ III and IV) $\mathrm{n}_{\sigma}(\mathbf{k} \pm \mathbf{q} / 2)=\mathrm{n}_{\sigma}(\mathbf{k}) \pm \frac{1}{2} \mathbf{q} \cdot \nabla_{\mathbf{k}} \mathrm{n}_{\sigma}(\mathbf{k})+\mathcal{O}\left(\|\mathbf{q}\|^{2}\right)$, for small $\|\mathbf{q}\|$, MCDC (1998) deduce from Eq. (14) (c.f. Eq. 63) below)

$$
\left(i q_{0}, \mathbf{q}\right)_{\nu} J^{\nu, \mathbf{k} \sigma}(q)=-\mathbf{q} \cdot \nabla_{\mathbf{k}} \mathrm{n}_{\sigma}(\mathbf{k})+\mathcal{O}\left(\|\mathbf{q}\|^{3}\right) .
$$

The screened interaction $D_{\mathbf{k} \mathbf{k}^{\prime}}^{\sigma \sigma^{\prime}}(q)$ in Eq. (2) is that within the random-phase approximation (RPA),

$$
D_{\mathbf{k} \mathbf{k}^{\prime}}^{\sigma \sigma^{\prime}}(q)=g_{\mathbf{k} \mathbf{k}^{\prime}}^{\sigma \sigma^{\prime}}(\mathbf{q})+\sum_{\sigma^{\prime \prime}} \int_{k^{\prime \prime}} g_{\mathbf{k} \mathbf{k}^{\prime \prime}}^{\sigma \sigma^{\prime \prime}}(\mathbf{q}) G\left(k^{\prime \prime}-q / 2\right) G\left(k^{\prime \prime}+q / 2\right) D_{\mathbf{k}^{\prime \prime} \mathbf{k}^{\prime}}^{\sigma^{\prime \prime} \sigma^{\prime}}(q)
$$

In practice, the interacting 'bubble' $G\left(k^{\prime \prime}-q / 2\right) G\left(k^{\prime \prime}+q / 2\right)$ is replaced by the non-interacting one (MCDC 1998), i.e. $G_{0}\left(k^{\prime \prime}-q / 2\right) G_{0}\left(k^{\prime \prime}+q / 2\right)$; this on account of the generalised CLT, under the assumption that $\mathbf{k}^{\prime \prime}$ is confined to a small neighbourhood of $\mathbf{k}_{F}$. For a comment on the generalised CLT in $d>1$ see (Farid 1999b).

From the equation of continuity $[\hat{n}(\mathbf{q}), \hat{H}]_{-}=\mathbf{q} \cdot \hat{\mathbf{j}}(\mathbf{q})$, where

$$
\hat{n}(\mathbf{q}):=\sum_{\sigma} \hat{n}_{\sigma}(\mathbf{q})
$$

(see Eq. (四) above), one can obtain the conserved current $\hat{\mathbf{j}}(\mathbf{q})$ for the system governed by the Hamiltonian $\hat{H}$ in Eq. (1). The conserved current $\hat{\mathbf{j}}_{0}(\mathbf{q})$ corresponding to $\hat{H}_{0}$ in Eq. (1) is obtained through application of the continuity equation $\left[\hat{n}(\mathbf{q}), \hat{H}_{0}\right]_{-}=\mathbf{q} \cdot \hat{\mathbf{j}}_{0}(\mathbf{q})$, 毛

$$
\hat{\mathbf{j}}_{0}(\mathbf{q})=\sum_{\mathbf{k} \sigma} \mathbf{v}_{\mathbf{k}}^{0} \hat{a}_{\mathbf{k}-\mathbf{q} / 2, \sigma}^{\dagger} \hat{a}_{\mathbf{k}+\mathbf{q} / 2, \sigma}
$$

where $\mathbf{v}_{\mathbf{k}}^{0}:=\nabla_{\mathbf{k}} \varepsilon_{\mathbf{k}}^{0} \equiv \mathbf{k}$. The commutator $\left[\hat{n}(\mathbf{q}), \hat{H}_{I}\right]_{-}$gives rise to 'non-diagonal' quartic contributions (involving four different momenta) to the current operator $\hat{\mathbf{j}}_{I}(\mathbf{q}):=\hat{\mathbf{j}}(\mathbf{q})-\hat{\mathbf{j}}_{0}(\mathbf{q})$, which are irrelevant in the low-energy limit (from the renormalisation-group perspective); taking the 'diagonal' contributions, corresponding to the momentum transfer $\mathbf{q}^{\prime}$ equal to either $\mathbf{q}$ or $-\mathbf{q}$, the following (approximate) expression is obtained (MCDC 1998)

$$
\begin{aligned}
{\left[\hat{n}(\mathbf{q}), \hat{H}_{I}\right]_{-} } & =\frac{1}{2 V} \sum_{\mathbf{k} \sigma} \sum_{\mathbf{k}^{\prime} \sigma^{\prime}} g_{\mathbf{k} \mathbf{k}^{\prime}}^{\sigma \sigma^{\prime}}(-\mathbf{q})\left[\hat{\mathrm{n}}_{\sigma}(\mathbf{k}-\mathbf{q} / 2)-\hat{\mathrm{n}}_{\sigma}(\mathbf{k}+\mathbf{q} / 2)\right] \hat{a}_{\mathbf{k}^{\prime}-\mathbf{q} / 2, \sigma^{\prime}}^{\dagger} \hat{a}_{\mathbf{k}^{\prime}+\mathbf{q} / 2, \sigma^{\prime}} \\
& +\frac{1}{2 V} \sum_{\mathbf{k} \sigma} \sum_{\mathbf{k}^{\prime} \sigma^{\prime}} g_{\mathbf{k} \mathbf{k}^{\prime}}^{\sigma \sigma^{\prime}}(\mathbf{q}) \hat{a}_{\mathbf{k}-\mathbf{q} / 2, \sigma}^{\dagger} \hat{a}_{\mathbf{k}+\mathbf{q} / 2, \sigma}\left[\hat{\mathbf{n}}_{\sigma^{\prime}}\left(\mathbf{k}^{\prime}-\mathbf{q} / 2\right)-\hat{\mathrm{n}}_{\sigma^{\prime}}\left(\mathbf{k}^{\prime}+\mathbf{q} / 2\right)\right]
\end{aligned}
$$

MCDC (1998) cast the right-hand side (RHS) of this expression into the form $\mathbf{q} \cdot \hat{\mathbf{j}}_{I}(\mathbf{q})$, with

$$
\hat{\mathbf{j}}_{I}(\mathbf{q})=\sum_{\mathbf{k} \sigma} \hat{\mathbf{v}}_{\mathbf{k} \sigma}^{I}(\mathbf{q}) \hat{a}_{\mathbf{k}-\mathbf{q} / 2, \sigma}^{\dagger} \hat{a}_{\mathbf{k}+\mathbf{q} / 2, \sigma}
$$

where

\footnotetext{
${ }^{9}$ Explicit calculation yields $\left[\hat{n}(\mathbf{q}), \hat{H}_{0}\right]_{-}=\sum_{\mathbf{k} \sigma}\left(\varepsilon_{\mathbf{k}+\mathbf{q} / 2}^{0}-\varepsilon_{\mathbf{k}-\mathbf{q} / 2}^{0}\right) \hat{a}_{\mathbf{k}-\mathbf{q} / 2, \sigma}^{\dagger} \hat{a}_{\mathbf{k}+\mathbf{q} / 2, \sigma}$. With $\varepsilon_{\mathbf{k}}^{0}=\|\mathbf{k}\|^{2} / 2$ (see Footnote 6 above and Footnote 12 below), one has $\varepsilon_{\mathbf{k}+\mathbf{q} / 2}^{0}-\varepsilon_{\mathbf{k}-\mathbf{q} / 2}^{0}=\mathbf{q} \cdot \mathbf{k} \equiv \mathbf{q} \cdot \mathbf{v}_{\mathbf{k}}^{0}$.
} 


$$
\hat{\mathbf{v}}_{\mathbf{k} \sigma}^{I}(\mathbf{q}):=-\frac{1}{V} \sum_{\mathbf{k}^{\prime} \sigma^{\prime}} g_{\mathbf{k} \mathbf{k}^{\prime}}^{\sigma \sigma^{\prime}}(\mathbf{q}) \nabla_{\mathbf{k}^{\prime}} \hat{\mathbf{n}}_{\sigma^{\prime}}\left(\mathbf{k}^{\prime}\right)
$$

In obtaining the result in Eq. (21), $\operatorname{MCDC}(1998)$ employ $\hat{\mathrm{n}}_{\sigma}(\mathbf{k}-\mathbf{q} / 2)-\hat{\mathrm{n}}_{\sigma}(\mathbf{k}+\mathbf{q} / 2)=\mathbf{q} \cdot\left[\boldsymbol{\nabla}_{\mathbf{k}} \hat{\mathbf{n}}_{\sigma}(\mathbf{k})\right]+\mathcal{O}\left(\|\mathbf{q}\|^{3}\right)$ (see $\S \S$ III.B and IV however; also compare with Eq. (63) below), and the same for $\hat{n}_{\sigma^{\prime}}\left(\mathbf{k}^{\prime}-\mathbf{q} / 2\right)-\hat{n}_{\sigma^{\prime}}\left(\mathbf{k}^{\prime}+\mathbf{q} / 2\right)$. On account of the fact that for the ground and low-lying excited states of the system $\left[\boldsymbol{\nabla}_{\mathbf{k}} \hat{\mathbf{n}}_{\sigma}(\mathbf{k})\right]$ is most significant for $\mathbf{k}=\mathbf{k}_{F}$ (the same concerning $\left[\boldsymbol{\nabla}_{\mathbf{k}^{\prime}} \hat{\mathbf{n}}_{\sigma^{\prime}}\left(\mathbf{k}^{\prime}\right)\right]$ for $\mathbf{k}^{\prime}=\mathbf{k}_{F}^{\prime}$ ), MCDC (1998) make use of the following relationship concerning the radial part of the $\mathbf{k}^{\prime}$ integration (that is that in the direction normal to the Fermi surface) in the expression for $\hat{\mathbf{v}}_{\mathbf{k} \sigma}^{I}(\mathbf{q})$ in Eq. (22), 10

$$
\begin{aligned}
\int \mathrm{d} k_{r}^{\prime} k_{r}^{\prime d-1} g_{\mathbf{k} \mathbf{k}^{\prime}}^{\sigma \sigma^{\prime}}(\mathbf{q}) \nabla_{\mathbf{k}^{\prime}} \hat{\mathbf{n}}_{\sigma^{\prime}}\left(\mathbf{k}^{\prime}\right) & \approx\left\|\mathbf{k}_{F}^{\prime}\right\|^{d-1} g_{\mathbf{k} \mathbf{k}_{F}^{\prime}}^{\sigma \sigma^{\prime}}(\mathbf{q}) \int \mathrm{d} k_{r}^{\prime} \nabla_{\mathbf{k}^{\prime}} \hat{\mathrm{n}}_{\sigma^{\prime}}\left(\mathbf{k}^{\prime}\right) \\
& =-\left\|\mathbf{k}_{F}^{\prime}\right\|^{d-1} g_{\mathbf{k} \mathbf{k}_{F}^{\prime}}^{\sigma \sigma^{\prime}}(\mathbf{q}) \boldsymbol{n}_{\mathbf{k}_{F}^{\prime}},
\end{aligned}
$$

where $\boldsymbol{n}_{\mathbf{k}_{F}^{\prime}}$ denotes the outward unit vector normal to the Fermi surface at $\mathbf{k}_{F}^{\prime}$. Here $d$ is not necessarily integer (see Appendix). In arriving at the last expression on the RHS of Eq. (23), MCDC (1998) have made use of the fact that for the ground and low-lying excited states of the system, $\hat{\mathbf{n}}_{\sigma^{\prime}}\left(\mathbf{k}^{\prime}\right)$ yields unity for $\mathbf{k}^{\prime}$ deep inside and zero for $\mathbf{k}^{\prime}$ far outside the Fermi sea (that is for $\mathbf{k}^{\prime}=\mathbf{k}_{i}^{\prime}$ and $\mathbf{k}^{\prime}=\mathbf{k}_{o}^{\prime}$, respectively). MCDC (1998) observe that the RHS of Eq. (23) (which is a $c$-number) would remain intact if $\hat{\mathbf{n}}_{\sigma^{\prime}}\left(\mathbf{k}^{\prime}\right)$ on the left-hand side (LHS) were the expectation value $\left\langle\hat{\mathrm{n}}_{\sigma^{\prime}}\left(\mathbf{k}^{\prime}\right)\right\rangle \equiv \mathrm{n}_{\sigma^{\prime}}\left(\mathbf{k}^{\prime}\right)$. 11 Thus MCDC (1998) employ (c.f. Eq. (22) above)

$$
\hat{\mathbf{v}}_{\mathbf{k} \sigma}^{I}(\mathbf{q}) \rightarrow \mathbf{v}_{\mathbf{k} \sigma}^{I}(\mathbf{q}):=-\frac{1}{V} \sum_{\mathbf{k}^{\prime} \sigma^{\prime}} g_{\mathbf{k} \mathbf{k}^{\prime}}^{\sigma \sigma^{\prime}}(\mathbf{q}) \nabla_{\mathbf{k}^{\prime}} \mathrm{n}_{\sigma^{\prime}}\left(\mathbf{k}^{\prime}\right),
$$

and consequently (c.f. Eq. (21) above)

$$
\hat{\mathbf{j}}_{I}(\mathbf{q}) \rightarrow \hat{\mathbf{j}}_{I}(\mathbf{q}):=\sum_{\mathbf{k} \sigma} \mathbf{v}_{\mathbf{k} \sigma}^{I}(\mathbf{q}) \hat{a}_{\mathbf{k}-\mathbf{q} / 2, \sigma}^{\dagger} \hat{a}_{\mathbf{k}+\mathbf{q} / 2, \sigma} .
$$

It is this $\hat{\mathbf{j}}_{I}(\mathbf{q})$ that MCDC (1998) employ in the construction of the generalised DL scheme. In $\S$ III.B we extensively discuss the shortcomings of the foundation of this expression for $\hat{\mathbf{j}}_{I}(\mathbf{q})$. Here we only briefly mention that, contrary to the statement by MCDC (1998), the relationship between the second and the third expression in Eq. (23)) is defective: momenta being confined to a narrow band of width $\lambda$ circumscribing the Fermi surface, we have $\left\|\mathbf{k}_{o}-\mathbf{k}_{i}\right\|=\lambda$. With $0<\lambda \ll\left\|\mathbf{k}_{F}\right\|$, unless the ground and low-lying excited states of the system be uncorrelated (or at most weakly correlated), it is not permitted to replace $\hat{\mathbf{n}}_{\sigma^{\prime}}\left(\mathbf{k}_{i}\right)$ by 1 and $\hat{\mathbf{n}}_{\sigma^{\prime}}\left(\mathbf{k}_{o}\right)$ by 0 . To clarify this point, let us take the expectation value of the expressions in Eq. (23) with respect to the $N$-particle ground state of $\hat{H}$. With $0<\lambda \ll\left\|\mathbf{k}_{F}^{\prime}\right\|$, it is seen that -1 on the RHS of Eq. (23) has to be replaced by $\mathrm{n}_{\sigma^{\prime}}\left(\mathbf{k}_{o}\right)-\mathrm{n}_{\sigma^{\prime}}\left(\mathbf{k}_{i}\right) \approx-Z_{\mathbf{k}_{F}^{\prime} \sigma^{\prime}}$, where $Z_{\mathbf{k}_{F}^{\prime} \sigma^{\prime}}$ stands for the amount of discontinuity in $\mathbf{n}_{\sigma^{\prime}}\left(\mathbf{k}^{\prime}\right)$ at $\mathbf{k}^{\prime}=\mathbf{k}_{F}^{\prime}$ which is less than unity for interacting systems and is equal to zero for such NFL states as the marginal (Varma, et al., 1989, 1990, Littlewood and Varma 1991) and Luttinger liquids (Haldane 1980, 1981, Anderson 1997). This undue replacement of $Z_{\mathbf{k}_{F}^{\prime}} \sigma^{\prime}$ by unity, is exactly the step undertaken in the construction of the Haldane (1992) bosonisation scheme in $d>1$; the consequences of this replacement would not be as severe were it not that in cases where $Z_{\mathbf{k}_{F}^{\prime}} \sigma^{\prime}$ is small, or vanishing, the other contributions, which are neglected within the bosonisation scheme, were not so significantly more dominant than that of the quantum anomaly that in an enhanced way (through replacing $Z_{\mathbf{k}_{F}^{\prime}} \sigma^{\prime}$ by unity) is taken into account (Farid 1999b). We observe that, unless the system under consideration be non-interacting, the constraint on $\lambda$, namely $\lambda \ll\left\|\mathbf{k}_{F}\right\|$, implies that in contradiction to the suggestion invoked by Eq. (23), $\int \mathrm{d} k_{r}^{\prime}{ }_{r}{k_{r}^{\prime}}_{r}^{d-1} g_{\mathbf{k} \mathbf{k}^{\prime}}^{\sigma \sigma^{\prime}}(\mathbf{q}) \nabla_{\mathbf{k}^{\prime}} \hat{\mathbf{n}}_{\sigma^{\prime}}\left(\mathbf{k}^{\prime}\right)$ is not even approximately a $c$-number. Be it as it may, as we shall demonstrate in $\S$ III.B, the expression in Eq. (21) with $\hat{\mathbf{v}}_{\mathbf{k} \sigma}^{I}(\mathbf{q})$ as defined in Eq. (22) is not the appropriate expression for $\hat{\mathbf{j}}_{I}(\mathbf{q})$ and, moreover, the appropriate expression (see Eqs. (51) and (49) below) even for large $\lambda$ cannot be replaced by a $c$-number.

Through applying $\left(i q_{0}, \mathbf{q}\right)_{\nu}$ to both sides of Eq. (10) and making use of Eqs. (14), (21) and (22), MCDC (1998) obtain the following crucial relationship

\footnotetext{
${ }^{10}$ In the thermodynamic limit one has $V^{-1} \sum_{\mathbf{k}^{\prime}}(\ldots) \rightarrow(2 \pi)^{-d} \int \mathrm{d}^{d} \mathbf{k}^{\prime}(\ldots)$. For $d$-dimensional integrations see Appendix.

${ }^{11}$ MCDC (1998) state namely "The $k_{r}^{\prime}$ integral over the operator expression thus yields a $c$-number, and the same $c$-number is obtained when replacing $\hat{\mathbf{n}}_{\sigma^{\prime}}\left(\mathbf{k}^{\prime}\right)$ by its expectation value."
} 


$$
\left(i q_{0}, \mathbf{q}\right)_{\nu} \Gamma_{\sigma}^{\nu}(p ; q)=\left(i q_{0}, \mathbf{q}\right)_{\nu} \Lambda_{\sigma}^{\nu}(p ; q)
$$

In arriving at this result, use has been made of the relations $(2 \pi)^{-1} \int \mathrm{d} k_{0}^{\prime} \hat{a}_{k^{\prime}-q / 2, \sigma^{\prime}}^{\dagger} \hat{a}_{k^{\prime}+q / 2, \sigma^{\prime}}=\hat{a}_{\mathbf{k}^{\prime}-\mathbf{q} / 2, \sigma^{\prime}}^{\dagger} \hat{a}_{\mathbf{k}^{\prime}+\mathbf{q} / 2, \sigma^{\prime}}$ and

$$
-\boldsymbol{\Lambda}_{\sigma}^{\prime}(p ; q)+\left\langle\hat{\mathbf{j}}_{I}(q) \hat{a}_{p-q / 2, \sigma} \hat{a}_{p+q / 2, \sigma}^{\dagger}\right\rangle_{\mathrm{tr}}^{\mathrm{irr}} \equiv\left\langle\hat{\mathbf{j}}_{0}(q) \hat{a}_{p-q / 2, \sigma} \hat{a}_{p+q / 2, \sigma}^{\dagger}\right\rangle_{\mathrm{tr}}^{\mathrm{irr}}=: \boldsymbol{\Lambda}_{\sigma}(p ; q) .
$$

From the exact Ward identity in Eq. (13) one finally obtains

$$
\left(i q_{0}, \mathbf{q}\right)_{\nu} \Lambda_{\sigma}^{\nu}(p ; q)=G_{\sigma}^{-1}(p+q / 2)-G_{\sigma}^{-1}(p-q / 2)
$$

which in combination with Eq. (7) yields (MCDC 1998)

$$
\Lambda_{\sigma^{\prime}, \sigma}^{0}(p ; q)=\delta_{\sigma^{\prime}, \sigma} \frac{G_{\sigma}^{-1}(p+q / 2)-G_{\sigma}^{-1}(p-q / 2)}{i q_{0}-\mathbf{q} \cdot \mathbf{v}_{\mathbf{p}}^{0}} .
$$

Eq. (2) in conjunction with Eqs. (17), (29) and the Dyson equation leads to a closed set of equations for the single-particle Green function $G_{\sigma}(p)$. One has

$$
G_{\sigma}(p)=G_{0 ; \sigma}(p)\left\{1-\int_{p^{\prime}} \frac{D_{\mathbf{p} \mathbf{p}^{\prime}}^{\sigma \sigma}\left(p-p^{\prime}\right)}{i\left(p-p_{0}\right)-\mathbf{v}_{\left[\mathbf{p}+\mathbf{p}^{\prime}\right] / 2}^{0} \cdot\left(\mathbf{p}-\mathbf{p}^{\prime}\right)} G_{\sigma}\left(p^{\prime}\right)+X_{\sigma}(p) G_{\sigma}(p)\right\},
$$

where $G_{0 ; \sigma}(p)$ denotes the non-interacting counterpart of $G_{\sigma}(p)$ and

$$
X_{\sigma}(p):=\int_{p^{\prime}} \frac{D_{\mathbf{p} \mathbf{p}^{\prime}}^{\sigma \sigma}\left(p-p^{\prime}\right)}{i\left(p-p_{0}\right)-\mathbf{v}_{\left[\mathbf{p}+\mathbf{p}^{\prime}\right] / 2}^{0} \cdot\left(\mathbf{p}-\mathbf{p}^{\prime}\right)} .
$$

In practice (MCDC 1998) $D_{\mathbf{p} \mathbf{p}^{\prime}}^{\sigma \sigma}\left(p-p^{\prime}\right)$ (see Eq. (17)) is approximated by $D_{\mathbf{p} \mathbf{p}}^{\sigma \sigma}\left(p-p^{\prime}\right)=: D_{\mathbf{p}}^{\sigma \sigma}\left(p-p^{\prime}\right)$.

For the case of an isotropic system, through replacing $\left\|\mathbf{v}_{\mathbf{k}}^{0}\right\|$ by $v_{F}^{0}$ and employing for small $\mathbf{q}:=\mathbf{p}-\mathbf{p}^{\prime}$ (c.f. the denominators of the expressions on the RHSs of Eqs. (30) and (31))

$$
\mathbf{v}_{\left[\mathbf{p}+\mathbf{p}^{\prime}\right] / 2}^{0} \cdot\left(\mathbf{p}-\mathbf{p}^{\prime}\right)=v_{F}^{0}\left(p_{r}-p_{r}^{\prime}\right)+\mathcal{O}\left(\|\mathbf{q}\|^{2}\right),
$$

where $p_{r}:=\|\mathbf{p}\|-\left\|\mathbf{k}_{F}\right\|, p_{r}^{\prime}:=\left\|\mathbf{p}^{\prime}\right\|-\left\|\mathbf{k}_{F}\right\|$, and for small $q=\left(i q_{0}, \mathbf{q}\right)$,

$$
D_{\mathbf{p}}^{\sigma \sigma}(q)=D\left(q_{r} / q_{0}, q_{t} / q_{0}\right),
$$

where $q_{r}:=\mathbf{q} \cdot \mathbf{p} /\|\mathbf{p}\|$ and $q_{t}:=\left(\|\mathbf{q}\|^{2}-q_{r}^{2}\right)^{1 / 2} \geq 0$, the radial and tangential components of $\mathbf{q}$, respectively, MCDC (1998) render the expression for $G_{\sigma}(p)$ in Eq. (30) suitable for treatment by the conventional method of dimensional continuation (see Appendix - we note in passing that here $q_{t}$ is the component of $\mathbf{q}$ inside the tangent space specified in Appendix). In this way MCDC (1998) establish that the cross-over dimension from Luttinger- to FL state is $d=1$; that is, the metallic state of the system under consideration is a FL (though not necessarily a conventional one) in $d>1$. This finding is in agreement with the $\epsilon$-expansion result by Ueda and Rice (1984) around $d=1$; according to these authors, in the $d=1+\epsilon$ fermion system the only weak-coupling fixed point is the FL one. As we shall demonstrate in $\S$ III.A, the expression in Eq. (29) whose denominator on the RHS is the origin of the denominators on the RHSs of the expressions in Eqs. (30) and (31) (which is further simplified in Eq. (32)), is not valid except in the strictly non-interacting case. In $\S$ III.A we further indicate that for NFLs, $\Sigma_{\sigma}\left(\mathbf{p} \pm \mathbf{q} / 2, \omega \pm \omega_{0} / 2\right)$ does not allow for an effective disentangling of $\mathbf{q}$ from $\mathbf{p}$ (as well as $\omega_{0}$ from $\omega$ ) and consequently for these systems the appropriate equation for $G_{\sigma}(p)$ does not accommodate terms involving contributions whose form would resemble the denominator of the integrands on the RHSs of Eqs. (30) and (31) which thus would be amenable to such reduction as in Eq. (32). Note that the expression in Eq. (32) is specific in that it does not involve a component of $\mathbf{p}^{\prime}$ in the tangent subspace $S_{\perp}$ (see Appendix). Thus we conclude that the general formalism for systems of interacting fermions even in the limit of strong forward scattering does not lend itself for treatment by the conventional method of dimensional continuation.

In the following Section we subject the generalised DL formalism to a critical analysis and demonstrate its failure in $d>1$. We also indicate the underlying reasons for the exactness of this formalism in $d=1$ when applied to the one-dimensional Luttinger model (1963). 


\section{CRITICAL ANALYSES}

In this Section we present our analyses of some crucial elements that are involved in the derivation of the generalised DL method described in $\S$ II.

\section{A. On the relationship between $\Lambda_{\sigma}^{0}(p ; q)$ and $\Lambda_{\sigma}(p ; q)$}

Here we restrict our considerations to the case of zero temperature $(T=0)$ and deal with the analytic continuations of functions of imaginary energy along the real energy axis. We denote the analytic continuations of such functions as $G_{\sigma}(p)$ and $\Sigma_{\sigma}(p)$ by $G_{\sigma}(\mathbf{p}, \omega)$ and $\Sigma_{\sigma}(\mathbf{p}, \omega)$ respectively.

From the Dyson equation for the Green function it follows that 12

$$
\begin{aligned}
G_{\sigma}^{-1}(\mathbf{p}+ & \left.\mathbf{q} / 2, \omega+\omega_{0} / 2\right)-G_{\sigma}^{-1}\left(\mathbf{p}-\mathbf{q} / 2, \omega-\omega_{0} / 2\right)=\omega_{0}-\mathbf{q} \cdot \mathbf{v}_{\mathbf{p} \sigma}^{0} \\
- & {\left[\Sigma_{\sigma}\left(\mathbf{p}+\mathbf{q} / 2, \omega+\omega_{0} / 2\right)-\Sigma_{\sigma}\left(\mathbf{p}-\mathbf{q} / 2, \omega-\omega_{0} / 2\right)\right] . }
\end{aligned}
$$

Assuming continuous differentiability of $\Sigma_{\sigma}\left(\mathbf{p}^{\prime}, \omega^{\prime}\right)$ with respect to $\mathbf{p}^{\prime}$ and $\omega^{\prime}$ in neighbourhoods of $\mathbf{p}^{\prime}=\mathbf{p}$ and $\omega^{\prime}=\omega$ respectively, for sufficiently small $\|\mathbf{q}\|$ and $\left|\omega_{0}\right|$ we have

$$
\begin{array}{r}
G_{\sigma}^{-1}\left(\mathbf{p}+\mathbf{q} / 2, \omega+\omega_{0} / 2\right)-G_{\sigma}^{-1}\left(\mathbf{p}-\mathbf{q} / 2, \omega-\omega_{0} / 2\right)=\omega_{0}\left[1-\partial \Sigma_{\sigma}(\mathbf{p}, \omega) / \partial \omega\right] \\
-\mathbf{q} \cdot\left[\mathbf{v}_{\mathbf{p} \sigma}^{0}+\nabla_{\mathbf{p}} \Sigma_{\sigma}(\mathbf{p}, \omega)\right]+\mathcal{O}\left(\|\mathbf{q}\|^{\gamma},\left|\omega_{0}\right|^{\gamma^{\prime}}\right)
\end{array}
$$

where $\gamma, \gamma^{\prime}>1$. From Eqs. (28) and (35), up to corrections of the form $\mathcal{O}\left(\|\mathbf{q}\|^{\gamma},\left|\omega_{0}\right|^{\gamma^{\prime}}\right)$, we deduce

$$
\Lambda_{\sigma}^{0}(\mathbf{p}, \omega)=1-\frac{\partial \Sigma_{\sigma}(\mathbf{p}, \omega)}{\partial \omega}, \quad \boldsymbol{\Lambda}_{\sigma}(\mathbf{p}, \omega)=\mathbf{v}_{\mathbf{p} \sigma}^{0}+\nabla_{\mathbf{p}} \Sigma_{\sigma}(\mathbf{p}, \omega)
$$

Following the expressions in Eq. (6) relating $\Lambda_{\sigma}^{0}$ to $\boldsymbol{\Lambda}_{\sigma}$, the results in Eq. (36) imply

$$
\nabla_{\mathbf{p}} \Sigma_{\sigma}(\mathbf{p}, \omega)=-\frac{\partial \Sigma_{\sigma}(\mathbf{p}, \omega)}{\partial \omega} \mathbf{v}_{\mathbf{p} \sigma}^{0}
$$

which must hold exactly for $\mathbf{p}=\mathbf{p}_{F}$ and $\omega=\omega_{F}$ if the results in Eqs. (7) and (29) are to be asymptotically valid for $\|\mathbf{q}\| \rightarrow 0$ and $\left|\omega_{0}\right| \rightarrow 0$. We note that a $\Sigma_{\sigma}(\mathbf{p}, \omega)$ whose associated $\Sigma_{\sigma}\left(\mathbf{p}_{F}, \omega\right)$ and $\Sigma_{\sigma}\left(\mathbf{p}, \omega_{F}\right)$ are continuously differentiable functions of $\omega$ and $\mathbf{p}$ in neighbourhoods of $\omega=\omega_{F}$ and $\mathbf{p}=\mathbf{p}_{F}$, respectively, by definition corresponds to a FL (Farid 1999a). In other words, if valid, Eq. (37) must hold for FLs when $\omega=\omega_{F}$ as $\mathbf{p} \rightarrow \mathbf{p}_{F}$ and $\mathbf{p}=\mathbf{p}_{F}$ as $\omega \rightarrow \omega_{F}$.

Let us now consider the following asymptotic expressions which apply to conventional FLs in $d=2$ for $\mathbf{p}$ close to the Fermi surface and $\omega \rightarrow \omega_{F}$ (Hodges, Smith and Wilkins 1971, Bloom 1975) 13 (in what follows we assume the system under consideration to be in the paramagnetic phase and thus suppress the spin indices):

$$
\begin{aligned}
& \operatorname{Re} \Sigma(\mathbf{p}, \omega) \sim \Sigma\left(\mathbf{p}, \omega_{F}\right)+\beta_{\mathbf{p}}\left(\omega-\omega_{F}\right), \\
& \operatorname{Im} \Sigma(\mathbf{p}, \omega) \sim \alpha_{\mathbf{p}} \operatorname{sgn}\left(\omega-\omega_{F}\right)\left(\omega-\omega_{F}\right)^{2} \ln \left|\omega-\omega_{F}\right|,
\end{aligned}
$$

where $\beta_{\mathbf{p}}$ and $\alpha_{\mathbf{p}}$ are real valued and for interacting FLs $\beta_{\mathbf{p}}<0$ (strictly negative; see text following Eq. (44) below) and $\alpha_{\mathbf{p}} \geq 0$; for conventional FLs in $d=2, \Sigma\left(\mathbf{p}, \omega_{F}\right) \not \equiv 0$ and $\alpha_{\mathbf{p}}>0$. Substitution of the RHSs of Eqs. (38) and (39) in Eq. (37) results in

\footnotetext{
${ }^{12}$ In expressions akin to that in Eq. (34) (such as that presented in Eq. 42 below), MCDC (1998) add $\mathcal{O}\left(\|\mathbf{q}\|^{2}\right)$ to the RHSs in order to account for the fact that in general the non-interacting energy dispersions can be more complicated than linear or quadratic functions of momentum. We do not follow this practice for the reason that the one-particle kinetic-energy operator $\hat{H}_{0}$ and the velocity operator $\hat{\mathbf{v}}$ are related to one another through the momentum operator $\hat{\mathbf{p}} ;$ we have $\hat{H}_{0}:=\hat{\mathbf{p}}^{2} / 2$ and $\hat{\mathbf{v}}:=\hat{\mathbf{p}}$ (we use $m_{f}=1$ throughout), so that in the momentum representation must hold (assuming a uniform state) $\varepsilon_{\mathbf{p}}^{0} \equiv\|\mathbf{p}\|^{2} / 2$ and $\mathbf{v}_{\mathbf{p}}^{0} \equiv \mathbf{p}$ for all $\mathbf{p}$ (from this perspective $\varepsilon_{\mathbf{p}+\mathbf{q} / 2}^{0}-\varepsilon_{\mathbf{p}-\mathbf{q} / 2}^{0}=\mathbf{q} \cdot \mathbf{v}_{\mathbf{p}}^{0}$, whence absence of $\mathcal{O}\left(\|\mathbf{q}\|^{2}\right.$ ) in Eq. (42)). A fully consistent generalisation of the formalism considered in the present work, that is one capable of dealing with general non-interacting energy dispersions, must therefore involve some reformulation of the problem at hand.

13 See also Fujimoto (1990) and Fukuyama, Narikio and Hasegawa (1991).
} 


$$
\begin{aligned}
\boldsymbol{\nabla}_{\mathbf{p}} \Sigma\left(\mathbf{p}, \omega_{F}\right)+\left[\boldsymbol{\nabla}_{\mathbf{p}} \beta_{\mathbf{p}}\right]\left(\omega-\omega_{F}\right) & \sim-\beta_{\mathbf{p}} \mathbf{v}_{\mathbf{p}}^{0}, \\
{\left[\boldsymbol{\nabla}_{\mathbf{p}} \alpha_{\mathbf{p}}\right]\left(\omega-\omega_{F}\right) \ln \left|\omega-\omega_{F}\right| } & \sim-\left(2 \ln \left|\omega-\omega_{F}\right|+1\right) \alpha_{\mathbf{p}} \mathbf{v}_{\mathbf{p}}^{0} .
\end{aligned}
$$

We now consider the consequences of these results. As will become evident, Eqs. (40) and (41) hold only for strictly non-interacting FLs (in this case they hold identically), thus implying incorrectness of Eq. (37) and by extension that of the expressions in Eq. (6) for interacting systems. For $\omega=\omega_{F}$, Eq. (40) leads to $\nabla_{\mathbf{p}} \Sigma\left(\mathbf{p}, \omega_{F}\right) \equiv-\beta_{\mathbf{p}} \mathbf{v}_{\mathbf{p}}^{0}$ (for $\mathbf{p}$ in the vicinity of the Fermi momentum), which, according to Eq. (40) for $\omega \neq \omega_{F}$, implies $\nabla_{\mathbf{p}} \beta_{\mathbf{p}} \equiv \mathbf{0} \Longleftrightarrow \beta_{\mathbf{p}} \equiv 0$ (see further on). This result in turn necessitates $\nabla_{\mathbf{p}} \Sigma\left(\mathbf{p}, \omega_{F}\right) \equiv \mathbf{0}$; further, for $\omega \rightarrow \omega_{F}$ the LHS of Eq. (41) vanishes while, unless $\alpha_{\mathbf{p}} \equiv 0$, the RHS diverges. It follows that unless the system of fermions (in $d=2$ ) be non-interacting, Eq. (37) and, in consequence, the relations in Eq. (6) are incorrect (see text following Eq. (45) below). For non-interacting systems we have namely (see Footnote 12 above)

$$
G_{0}^{-1}\left(\mathbf{p}+\mathbf{q} / 2, \omega+\omega_{0} / 2\right)-G_{0}^{-1}\left(\mathbf{p}-\mathbf{q} / 2, \omega-\omega_{0} / 2\right) \equiv \omega_{0}-\mathbf{q} \cdot \mathbf{v}_{\mathbf{p}}^{0},
$$

from which it is readily deduced that, with $\left(1, \mathbf{v}_{\mathbf{p}}^{0}\right)^{\nu}$ the non-interacting counterpart of the irreducible vertex part $\Lambda_{\sigma}^{\nu}(p ; q)$, the relations in Eq. (6) are identically valid in the strictly non-interacting case. This completes the proof of our statement with regard to the condition for validity of the expressions in Eq. (6). We emphasise that the case of $d=1$ is very special when $\mathbf{v}_{\mathbf{p}}^{0}$ is a constant, say $\mathbf{v}^{0}$ (which points to a definite direction), as is the case for the one-dimensional Luttinger (1963) model. Here the relations in Eq. (6) hold identically, for whatever $\Lambda_{\sigma}^{0}(p ; q)$ may be, from the definition for $\hat{j}_{0}^{\nu}(\mathbf{q})$, namely (see Eqs. (18), (1) and (19) above)

$$
\hat{j}_{0}^{\nu}(\mathbf{q}):=\left(\hat{n}(\mathbf{q}), \hat{\mathbf{j}}_{0}(\mathbf{q})\right)^{\nu} \equiv \sum_{\mathbf{k} \sigma}\left(1, \mathbf{v}_{\mathbf{k}}^{0}\right)^{\nu} \hat{a}_{\mathbf{k}-\mathbf{q} / 2 \sigma}^{\dagger} \hat{a}_{\mathbf{k}+\mathbf{q} / 2 \sigma},
$$

it follows that $\boldsymbol{\Lambda}_{\sigma}(p ; q)$ is $\mathbf{v}^{0}$ times $\Lambda_{\sigma}^{0}(p ; q)$ (see Eq. (6)).

We conclude that the relations in Eq. (6) are not asymptotically correct in the forward-scattering limit when $d>1$, unless the system under consideration be non-interacting, in which case they are identically valid in any spatial dimension.

Above we have asserted that $\boldsymbol{\nabla}_{\mathbf{p}} \beta_{\mathbf{p}} \equiv \mathbf{0} \Longleftrightarrow \beta_{\mathbf{p}} \equiv 0$. We now demonstrate the validity of this equivalence. For FLs, it can be shown that (Farid 1999a)

$$
\beta_{\mathbf{p}}=\frac{1}{\pi} \int_{0}^{\infty} \frac{\mathrm{d} \omega^{\prime}}{\omega^{\prime 2}}\left[\operatorname{Im} \Sigma\left(\mathbf{p}, \omega^{\prime}+\omega_{F}\right)-\operatorname{Im} \Sigma\left(\mathbf{p},-\omega^{\prime}+\omega_{F}\right)\right] .
$$

We note in passing that since for interacting FLs the integrand of this expression is not identically vanishing and, moreover, owing to the assumed stability of the system $\operatorname{Im} \Sigma\left(\mathbf{p}, \omega^{\prime}+\omega_{F}\right)$ and $-\operatorname{Im} \Sigma\left(\mathbf{p},-\omega^{\prime}+\omega_{F}\right)$ are never positive for $\omega^{\prime} \geq 0$, it follows that $\beta_{\mathbf{p}}<0$ (strictly negative). The expression in Eq. (44) can be transformed into

$$
\beta_{\mathbf{p}}=\frac{1}{\pi} \operatorname{Im} \int_{\mu+0^{+}}^{i \infty} \frac{\mathrm{d} z}{(z-\mu)^{2}}[\Sigma(\mathbf{p}, z)-\Sigma(\mathbf{p}, 2 \mu-z)],
$$

where $\mu \in\left(\mu_{N}, \mu_{N+1}\right)$ with $\mu_{N}:=E_{N, 0}-E_{N-1,0} \equiv \omega_{F}$ and $\mu_{N+1}:=E_{N+1,0}-E_{N, 0}$. Here $E_{M, 0}$ stands for the total energy of the interacting $M$-particle ground state and $N$ denotes the actual number of the particles in the system. The $z$-integration in Eq. (45) is carried out along an arbitrary contour in the upper half of the complex $z$ plane, connecting $z=\mu+0^{+}$with the point of infinity. The arbitrariness (under the mentioned constraints) of the contour of the $z$ integration has a far-reaching consequence: assuming differentiability of $\beta_{\mathbf{p}}$ with respect to $\mathbf{p}$ (in some neighbourhood of $\left.\mathbf{p}_{F}\right)$, it follows from $\boldsymbol{\nabla}_{\mathbf{p}} \beta_{\mathbf{p}} \equiv \mathbf{0}$ that $\boldsymbol{\nabla}_{\mathbf{p}}[\Sigma(\mathbf{p}, z)-\Sigma(\mathbf{p}, 2 \mu-z)] \equiv \mathbf{0}$ for all $z$ in the upper half of the complex $z$ plane; by the Riemann-Schwarz reflection property with respect to the real axis, this identity holds also for all points in the lower half of the complex $z$ plane. 15 This implies that $\Sigma(\mathbf{p}, z) \equiv \Sigma(\mathbf{p}, 2 \mu-z)$ over the entire complex plane.

\footnotetext{
${ }^{14}$ For FLs $\int_{\mu}^{i \infty} \mathrm{d} z(z-\mu)^{-2} \operatorname{Im}[\Sigma(\mathbf{p}, z)-\Sigma(\mathbf{p}, 2 \mu-z)]$ exists, however $\int_{\mu}^{i \infty} \mathrm{d} z(z-\mu)^{-2}[\Sigma(\mathbf{p}, z)-\Sigma(\mathbf{p}, 2 \mu-z)]$ does not. Addition of the infinitesimal $0^{+}$to $\mu$ in the lower boundary of the latter integral (see Eq. (45)) formally renders this integral existent without affecting the value of $\beta_{\mathbf{p}}$.

${ }^{15}$ For $\operatorname{Im}(z) \neq 0, \Sigma(\mathbf{p}, z)$ satisfies the Riemann-Schwarz reflection property $\Sigma\left(\mathbf{p}, z^{*}\right) \equiv \Sigma^{*}(\mathbf{p}, z)$ (see e.g., DuBois 1959 , Luttinger 1961, Farid 1999a).
} 
In view of the expressions in Eqs. (38) and (39), this result is seen to apply only if $\Sigma$ (p, $z$ ) is independent of $z$, a property which is specific to non-interacting systems. A corollary to this result is that $\beta_{\mathbf{p}} \equiv 0$ (that is zero rather than any other constant that $\boldsymbol{\nabla}_{\mathbf{p}} \beta_{\mathbf{p}} \equiv \mathbf{0}$ would suggest in the first instance; see the text following Eq. (41) above). ${ }^{16}$

Our above analysis has explicitly concerned FLs (through our explicit use of the relations in Eqs. (38) and (39), but more importantly Eq. (44)), however, as can be easily verified, for NFLs the RHS of Eq. (34) does not allow for any effective separation of $\mathbf{q}$ and $\omega_{0}$ from $\mathbf{p}$ and $\omega$ respectively, so that in $d>1$ the relations in Eq. (6) lack even the most fundamental basis for validity as regards NFLs.

\section{B. On the relationship between $\Lambda_{\sigma}^{\nu}(p ; q)$ and $\Gamma_{\sigma}^{\nu}(p ; q)$}

Evidently for obtaining the $\hat{\mathbf{j}}_{I}(\mathbf{q})$ as presented in Eq. (21) in terms of $\hat{\mathbf{v}}_{\mathbf{k} \sigma}^{I}(\mathbf{q})$ in Eq. (22), the functions in the square brackets on the RHS of Eq. (20) have been expanded to linear order in q. Such an expansion is in general invalid for reasons that we discuss in some detail in $\S$ IV. A second step involved in the derivation of $\hat{\mathbf{j}}_{I}(\mathbf{q})$ in Eq. (25) is that of replacing $\boldsymbol{\nabla}_{\mathbf{k}} \hat{\mathrm{n}}_{\sigma}(\mathbf{k})$ by the expectation value $\boldsymbol{\nabla}_{\mathbf{k}}\left\langle\hat{\mathbf{n}}_{\sigma}(\mathbf{k})\right\rangle$. The justification that has been given by MCDC (1998) for this substitution is untenable (in particular in $d>1$ ) (see also text following Eq. (25) above). For obtaining a generally valid expression for $\hat{\mathbf{j}}_{I}(\mathbf{q})$, the RHS of Eq. (20) must be first written in terms of four separate contributions, each involving only one of $\hat{\mathrm{n}}_{\sigma}(\mathbf{k}-\mathbf{q} / 2), \hat{\mathrm{n}}_{\sigma}(\mathbf{k}+\mathbf{q} / 2), \hat{\mathrm{n}}_{\sigma^{\prime}}\left(\mathbf{k}^{\prime}-\mathbf{q} / 2\right)$ and $\hat{\mathrm{n}}_{\sigma^{\prime}}\left(\mathbf{k}^{\prime}+\mathbf{q} / 2\right)$. Subsequently, in each of these contributions, by appropriate transformations of the summation variables $\mathbf{k}$ and $\mathbf{k}^{\prime}$, the vector $\mathbf{q}$ in the arguments of these functions must be transferred into that of the interaction function $g_{\mathbf{k} \mathbf{k}^{\prime}}^{\sigma \sigma^{\prime}}(\mp \mathbf{q})$, which we assume to be a continuously differentiable function of its indices $\mathbf{k}$ and $\mathbf{k}^{\prime}$ (c.f. statement preceding Eq. (7) in Castellani, Di Castro and Metzner 1994). In this way one obtains (for small $\|\mathbf{q}\|$ )

$$
\begin{aligned}
\hat{\mathbf{j}}_{I}(\mathbf{q})=\frac{1}{2 V} \sum_{\mathbf{k} \sigma} \sum_{\mathbf{k}^{\prime} \sigma^{\prime}}\{ & {\left[\nabla_{\mathbf{k}} g_{\mathbf{k} \mathbf{k}^{\prime}}^{\sigma \sigma^{\prime}}(-\mathbf{q})\right] \hat{\mathrm{n}}_{\sigma}(\mathbf{k}) \hat{a}_{\mathbf{k}^{\prime}-\mathbf{q} / 2, \sigma^{\prime}}^{\dagger} \hat{a}_{\mathbf{k}^{\prime}+\mathbf{q} / 2, \sigma^{\prime}} } \\
+ & {\left.\left[\boldsymbol{\nabla}_{\mathbf{k}^{\prime}} g_{\mathbf{k} \mathbf{k}^{\prime}}^{\sigma \sigma^{\prime}}(\mathbf{q})\right] \hat{a}_{\mathbf{k}-\mathbf{q} / 2, \sigma}^{\dagger} \hat{a}_{\mathbf{k}+\mathbf{q} / 2, \sigma} \hat{\mathrm{n}}_{\sigma^{\prime}}\left(\mathbf{k}^{\prime}\right)\right\} . }
\end{aligned}
$$

Making use of the commutation relation

$$
\left[\hat{a}_{\mathbf{k}-\mathbf{q} / 2, \sigma}^{\dagger} \hat{a}_{\mathbf{k}+\mathbf{q} / 2, \sigma}, \hat{\mathbf{n}}_{\sigma^{\prime}}\left(\mathbf{k}^{\prime}\right)\right]_{-}=\delta_{\sigma, \sigma^{\prime}}\left\{\delta_{\mathbf{k}+\mathbf{q} / 2, \mathbf{k}^{\prime}}-\delta_{\mathbf{k}-\mathbf{q} / 2, \mathbf{k}^{\prime}}\right\} \hat{a}_{\mathbf{k}-\mathbf{q} / 2, \sigma}^{\dagger} \hat{a}_{\mathbf{k}+\mathbf{q} / 2, \sigma},
$$

which is obtained through a repeated application of the canonical anti-commutation relations for $\hat{a}_{\mathbf{k}, \sigma}^{\dagger}$ and $\hat{a}_{\mathbf{k}, \sigma}$, the expression in Eq. (46) can be written as

$$
\begin{aligned}
\hat{\mathbf{j}}_{I}(\mathbf{q})= & \sum_{\mathbf{k} \sigma} \hat{\mathbf{v}}_{\mathbf{k} \sigma}^{I}(\mathbf{q}) \hat{a}_{\mathbf{k}-\mathbf{q} / 2, \sigma}^{\dagger} \hat{a}_{\mathbf{k}+\mathbf{q} / 2, \sigma} \\
& +\frac{1}{2 V} \sum_{\mathbf{k} \sigma}\left\{\left[\left.\nabla_{\mathbf{k}^{\prime}} g_{\mathbf{k} \mathbf{k}^{\prime}}^{\sigma \sigma}(\mathbf{q})\right|_{\mathbf{k}^{\prime}=\mathbf{k}+\mathbf{q} / 2}\right]-\left[\left.\boldsymbol{\nabla}_{\mathbf{k}^{\prime}} g_{\mathbf{k}_{\mathbf{k}}^{\prime}}^{\sigma \sigma}(\mathbf{q})\right|_{\mathbf{k}^{\prime}=\mathbf{k}-\mathbf{q} / 2}\right]\right\} \hat{a}_{\mathbf{k}-\mathbf{q} / 2, \sigma}^{\dagger} \hat{a}_{\mathbf{k}+\mathbf{q} / 2, \sigma}
\end{aligned}
$$

where (c.f. Eq. (22) above)

$$
\hat{\mathbf{v}}_{\mathbf{k} \sigma}^{I}(\mathbf{q}):=\frac{1}{V} \sum_{\mathbf{k}^{\prime} \sigma^{\prime}}\left[\nabla_{\mathbf{k}^{\prime}} \bar{g}_{\mathbf{k} \mathbf{k}^{\prime}}^{\sigma \sigma^{\prime}}(\mathbf{q})\right] \hat{\mathbf{n}}_{\sigma^{\prime}}\left(\mathbf{k}^{\prime}\right)
$$

in which

$$
\bar{g}_{\mathbf{k} \mathbf{k}^{\prime}}^{\sigma \sigma^{\prime}}(\mathbf{q}):=\frac{1}{2}\left(g_{\mathbf{k} \mathbf{k}^{\prime}}^{\sigma \sigma^{\prime}}(\mathbf{q})+g_{\mathbf{k}^{\prime} \mathbf{k}}^{\sigma^{\prime} \sigma}(-\mathbf{q})\right)
$$

\footnotetext{
${ }^{16}$ Let $\operatorname{Re}(z)=: \omega>\mu$ and $\operatorname{Im}(z)=: \eta, \eta \downarrow 0$. For such a $z$ we have $\operatorname{Im} \Sigma(\mathbf{p}, z) \leq 0$ while $\operatorname{Im} \Sigma(\mathbf{p}, 2 \mu-z) \geq 0$ (validity of both of these relations is necessary for the stability of the system). Satisfaction of $\Sigma(\mathbf{p}, z) \equiv \Sigma(\mathbf{p}, 2 \mu-z)$, for all $z$, in this particular case would imply $\operatorname{Im} \Sigma(\mathbf{p}, \omega+i \eta) \equiv 0$ for all $\omega>\mu$; it similarly would imply $\operatorname{Im} \Sigma(\mathbf{p}, \omega-i \eta) \equiv 0$ for all $\omega<\mu$. By the Kramers-Krönig relation it follows that $\Sigma(\mathbf{p}, z)$ is independent of $z$. Note that in obtaining this result we have not relied upon the specific form of the expressions in Eqs. (38) and (39).
} 
By assuming $\boldsymbol{\nabla}_{\mathbf{k}^{\prime}} g_{\mathbf{k} \mathbf{k}^{\prime}}^{\sigma, \sigma}(\mathbf{q})$ to be a continuously-differentiable function of $\mathbf{k}^{\prime}$, the last term on the RHS of Eq. (48) is seen to be proportional to $\mathbf{q}$, so that in the limit of $\mathbf{q} \rightarrow \mathbf{0}$ it can be neglected in comparison with the first term. For small $\|\mathbf{q}\|$ we can thus write

$$
\hat{\mathbf{j}}_{I}(\mathbf{q})=\sum_{\mathbf{k} \sigma} \hat{\mathbf{v}}_{\mathbf{k} \sigma}^{I}(\mathbf{q}) \hat{a}_{\mathbf{k}-\mathbf{q} / 2, \sigma}^{\dagger} \hat{a}_{\mathbf{k}+\mathbf{q} / 2, \sigma}
$$

where $\hat{\mathbf{v}}_{\mathbf{k} \sigma}^{I}(\mathbf{q})$ is defined in Eq. (49). Note in passing, that for a constant interaction (i.e. a $\mathbf{k}, \mathbf{k}^{\prime}$-independent $g_{\mathbf{k}}^{\sigma \sigma^{\prime}}(\mathbf{q})$ ), $\hat{\mathbf{v}}_{\mathbf{k} \sigma}^{I}(\mathbf{q}) \equiv \hat{\mathbf{0}}$, so that Eq. (51) correctly yields $\hat{\mathbf{j}}_{I}(\mathbf{q}) \equiv \hat{\mathbf{0}}$, or $\hat{\mathbf{j}}(\mathbf{q}) \equiv \hat{\mathbf{j}}_{0}(\mathbf{q})$.

The correlated velocity operator in Eq. (49) is distinct from that in Eq. (22). To bring out the fundamental difference between the two, let us follow MCDC (1998) and replace the operator $\hat{\mathbf{n}}_{\sigma^{\prime}}\left(\mathbf{k}^{\prime}\right)$ on the RHS of Eq. (49) by its expectation value $\mathbf{n}_{\sigma^{\prime}}\left(\mathbf{k}^{\prime}\right)$ and compare the resulting vector $\mathbf{v}_{\mathbf{k} \sigma}^{I}(\mathbf{q})$ with that in Eq. (24). Since by assumption $\nabla_{\mathbf{k}^{\prime}} \bar{g}_{\mathbf{k}}^{\sigma \sigma^{\prime}}(\mathbf{q})$ is a smooth function of $\mathbf{k}^{\prime}$ (see text preceding Eq. (46) above), we can write (recall that $\left\|\mathbf{k}_{o}^{\prime}-\mathbf{k}_{i}^{\prime}\right\|=\lambda \ll\left\|\mathbf{k}_{F}^{\prime}\right\|$ )

$$
\int \mathrm{d} k_{r}^{\prime} k_{r}^{\prime d-1}\left[\boldsymbol{\nabla}_{\mathbf{k}^{\prime}} \bar{g}_{\mathbf{k} \mathbf{k}^{\prime}}^{\sigma \sigma^{\prime}}(\mathbf{q})\right] \mathrm{n}_{\sigma^{\prime}}\left(\mathbf{k}^{\prime}\right) \approx\left[\left.\boldsymbol{\nabla}_{\mathbf{k}^{\prime}} \bar{g}_{\mathbf{k} \mathbf{k}^{\prime}}^{\sigma \sigma^{\prime}}(\mathbf{q})\right|_{\mathbf{k}^{\prime}=\mathbf{k}_{F}^{\prime}}\right] \int \mathrm{d} k_{r}^{\prime} k_{r}^{\prime d-1} \mathbf{n}_{\sigma^{\prime}}\left(\mathbf{k}^{\prime}\right)
$$

For the non-interacting case, where $\mathrm{n}_{\sigma^{\prime}}\left(\mathbf{k}^{\prime}\right)=\Theta\left(\mathbf{k}_{F}^{\prime}-\mathbf{k}^{\prime}\right.$ ) (recall that distances are measured along $\boldsymbol{n}_{\mathbf{k}_{F}^{\prime}}$ ), the integral on the RHS of Eq. (52) is equal to $\left(\left\|\mathbf{k}_{F}^{\prime}\right\|^{d}-\left\|\mathbf{k}_{i}^{\prime}\right\|^{d}\right) / d$, so that the RHS of Eq. (52) becomes equal to $\left[\left.\boldsymbol{\nabla}_{\mathbf{k}^{\prime}} \bar{g}_{\mathbf{k} \mathbf{k}^{\prime}}^{\sigma \sigma^{\prime}}(\mathbf{q})\right|_{\mathbf{k}^{\prime}=\mathbf{k}_{F}^{\prime}}\right]\left(\left\|\mathbf{k}_{F}^{\prime}\right\|^{d}-\left\|\mathbf{k}_{i}^{\prime}\right\|^{d}\right) / d$, to be compared with the RHS of Eq. 23), which is equally applicable to the non-interacting ground states, namely $-g_{\mathbf{k} \mathbf{k}_{F}^{\prime}}^{\sigma \sigma^{\prime}}(\mathbf{q})\left\|\mathbf{k}_{F}^{\prime}\right\|^{d} \boldsymbol{n}_{\mathbf{k}_{F}^{\prime}}$. The two results are manifestly different. This demonstrates that Eq. (24), and therefore Eq. (25), is fundamentally incorrect. This aspect is additional to the shortcoming of the result in Eq. (23), which as we have discussed in the text following Eq. (25), shows up when the ground and low-lying excited states of the system are (strongly) correlated. Thus we have unequivocally established that not only the change in Eq. (25), corresponding to replacing $\hat{\mathbf{v}}_{\mathbf{k} \sigma}^{I}(\mathbf{q})$ as defined in Eq. (22) by $\mathbf{v}_{\mathbf{k} \sigma}^{I}(\mathbf{q})$ as defined in Eq. (24), lacks justification (unless the system under consideration be non-interacting), but also that the expression for $\hat{\mathbf{v}}_{\mathbf{k} \sigma}^{I}(\mathbf{q})$ as presented in Eq. (22) (to be compared with the $\hat{\mathbf{v}}_{\mathbf{k} \sigma}^{I}(\mathbf{q})$ in Eq. (49)) is invalid, this on account of the fact that it relies on the Taylor expansion of an operator (that is $\hat{\mathbf{n}}_{\sigma}(\mathbf{k} \pm \mathbf{q} / 2)$ ) whose, in particular, expectation values with respect to ground states are in general non-differentiable (see $\S$ IV).

A major consequence of use of Eq. (51), or Eq. (21), rather than one in which the operator $\hat{\mathbf{v}}_{\mathbf{k} \sigma}^{I}(\mathbf{q})$ is replaced by the $c$-number $\mathbf{v}_{\mathbf{k} \sigma}^{I}(\mathbf{q})$ (effected through substituting $\mathbf{n}_{\sigma^{\prime}}\left(\mathbf{k}^{\prime}\right)$ for $\hat{\mathbf{n}}_{\sigma^{\prime}}\left(\mathbf{k}^{\prime}\right)$ in Eq. (49), or Eq. (22)), is violation of Eq. (26) which is the crucial link between the exact Ward identity in Eq. (13) and the expression in Eq. (28). To make this statement explicit, we first apply $\left(i q_{o}, \mathbf{q}\right)_{\nu}$ to both sides of Eq. (10) and subsequently substitute the expression on the RHS of Eq. (14) for $\left(i q_{0}, \mathbf{q}\right)_{\nu} J^{\nu, \mathbf{k}^{\prime} \sigma^{\prime}}(q)$. Note that in doing so, we depart from the approach by MCDC (1998) who employ the expression on the RHS of Eq. (16). Next, we write the expression for $\left(i q_{0}, \mathbf{q}\right)_{\nu} J^{\nu, \mathbf{k}^{\prime} \sigma^{\prime}}(q)$ in the contribution corresponding to $\left(i q_{0}, \mathbf{q}\right)_{\nu} \Gamma_{\sigma}^{\nu}(p ; q)$ as two separate terms and in each of these by transformations of the dummy variables of summation, remove $\mathbf{q} / 2$ from arguments of $n_{\sigma^{\prime}}\left(\mathbf{k}^{\prime}-\mathbf{q} / 2\right)$ and $n_{\sigma^{\prime}}\left(\mathbf{k}^{\prime}+\mathbf{q} / 2\right)$. This procedure is exactly the same as that which we employed for deducing $\hat{\mathbf{j}}_{I}(\mathbf{q})$ from the equation of continuity associated with $\hat{H}_{I}$ (see Eq. (46) above and text following it). Thus we obtain

$$
\begin{aligned}
& \frac{1}{V} \sum_{\mathbf{k}^{\prime} \sigma^{\prime} \mathbf{k}^{\prime \prime} \sigma^{\prime \prime}}\left(i q_{0}, \mathbf{q}\right)_{\nu} J^{\nu, \mathbf{k}^{\prime} \sigma^{\prime}}(q) g_{\mathbf{k}^{\prime} \mathbf{k}^{\prime \prime}}^{\sigma^{\prime} \sigma^{\prime \prime}}(q) \Lambda_{\sigma}^{\mathbf{k}^{\prime \prime} \sigma^{\prime \prime}}(p ; q) \\
& =\mathbf{q} \cdot \sum_{\mathbf{k}^{\prime \prime} \sigma^{\prime \prime}}\left\{\frac{1}{V} \sum_{\mathbf{k}^{\prime} \sigma^{\prime}} \mathbf{n}_{\sigma^{\prime}}\left(\mathbf{k}^{\prime}\right)\left[\nabla_{\mathbf{k}^{\prime}} g_{\mathbf{k}^{\prime} \mathbf{k}^{\prime \prime}}^{\sigma^{\prime} \sigma^{\prime \prime}}(\mathbf{q})\right]\right\} \Lambda_{\sigma}^{\mathbf{k}^{\prime \prime} \sigma^{\prime \prime}}(p ; q)
\end{aligned}
$$

Neglecting the possible difference between $g_{\mathbf{k} \mathbf{k}^{\prime}}^{\sigma \sigma^{\prime}}(\mathbf{q})$ and $\bar{g}_{\mathbf{k} \mathbf{k}^{\prime}}^{\sigma \sigma^{\prime}}(\mathbf{q})$ (see Eq. (50) above), it is seen that the term enclosed by curly braces in Eq. (53) is equal to the expectation value of $\hat{\mathbf{v}}_{\mathbf{k} \sigma}^{I}(\mathbf{q})$ as presented in Eq. (49)). As we have extensively discussed above, there exists no justification, whatever, for $\mathbf{v}_{\mathbf{k} \sigma}^{I}(\mathbf{q})$ to be a good representation of $\hat{\mathbf{v}}_{\mathbf{k} \sigma}^{I}(\mathbf{q})$. If, as in the treatment by MCDC (1998), $\hat{\mathbf{v}}_{\mathbf{k} \sigma}^{I}(\mathbf{q})$ could be replaced by its expectation value, then making use of the defining expression for $\Lambda_{\sigma}^{\mathbf{k}^{\prime \prime} \sigma^{\prime \prime}}(p ; q)$ in Eq. (12) and the expression for $\hat{\mathbf{j}}_{I}(\mathbf{q})$ in Eq. (51) wherein $\hat{\mathbf{v}}_{\mathbf{k} \sigma}^{I}(\mathbf{q})$ is replaced by $\mathbf{v}_{\mathbf{k} \sigma}^{I}(\mathbf{q})$, the RHS of Eq. (53) would reduce to $\mathbf{q} \cdot \boldsymbol{\Lambda}_{\sigma}(p ; q)$. Adding this result to $\left(i q_{0}, \mathbf{q}\right)_{\nu} \boldsymbol{\Lambda}_{\sigma}^{\prime \nu}(p ; q)$ (see Eq. (10), to whose both sides $\left(i q_{0}, \mathbf{q}\right)_{\nu}$ is applied), one would arrive at Eq. (26). We emphasise that in arriving at Eq. (26) it is the substitution of $\hat{\mathbf{v}}_{\mathbf{k} \sigma}^{I}(\mathbf{q})$ by $\mathbf{v}_{\mathbf{k} \sigma}^{I}(\mathbf{q})$ that plays the vital role and not whether $\mathbf{v}_{\mathbf{k} \sigma}^{I}(\mathbf{q})$ is the expectation value of $\hat{\mathbf{v}}_{\mathbf{k} \sigma}^{I}(\mathbf{q})$ as defined in Eq. (22) or Eq. (49). However, it should be realised that contrary to the case of the $\hat{\mathbf{v}}_{\mathbf{k} \sigma}^{I}(\mathbf{q})$ in Eq. (22) whose replacement by its expectation value can be justified for non-interacting systems (and by extension, weakly interacting 
systems) (see $\S$ II, text following Eq. (25)), replacement of the $\hat{\mathbf{v}}_{\mathbf{k} \sigma}^{I}(\mathbf{q})$ in Eq. (49), which we have demonstrated to be the correct velocity operator, by its expectation value is not justified, not even for cases where the ground and low-lying excited states of the system are non-interacting (or weakly interacting). Of course, in the non-interacting case $g_{\mathbf{k ~ k}}^{\sigma \sigma^{\prime}}(\mathbf{q})$ is identically vanishing so that the problem associated with the inadmissibility of replacing $\hat{\mathbf{v}}_{\mathbf{k} \sigma}^{I}(\mathbf{q})$ by $\mathbf{v}_{\mathbf{k} \sigma}^{I}(\mathbf{q})$ does not arise in this limit (see Eq. (49) above).

Above we have demonstrated that in $d>1$, the trivial relationship between the vertex parts $\Lambda_{\sigma}^{\nu}(p ; q)$ and $\Gamma_{\sigma}^{\nu}(p ; q)$ as given in Eq. (26) is invalid and consequently no direct link exists between the exact Ward identity in Eq. (13) and the expression in Eq. (28). Further, our considerations in $\S$ III.A made evident that also Eq. (7) has no rightful place in the theory of interacting fermions in $d>1$.

\section{MATHEMATICAL TRANSGRESSIONS AND "BLESSINGS" OF INCORRECT ERROR ESTIMATION}

In this Section we illustrate how in $d=1$, specifically in dealing with the one-dimensional Luttinger (1963) model at $T=0$, a combination of transgressions against mathematical rules and uncritical neglect of truncation errors can give rise to outcomes which coincide with the exact results, thus creating the false impression that this approach were justified. The particular example that we consider in this Section makes explicit that specifically in $d>1$ the same procedure yields manifestly incorrect results. Our illustrative example involves the ground-state momentum distribution function $\mathrm{n}(\mathbf{k})$ which is singular at $\mathbf{k}=\mathbf{k}_{F}$ both in the case of FLs and NFLs.

We first explicitly deal with the one-dimensional Luttinger model for spin-less fermions (Luttinger 1963, Mattis and Lieb 1965), characterised by the anomalous dimension $\alpha, \alpha \in(0,1)$. We note in passing that for the one-dimensional Hubbard model $0<\alpha<1 / 8$, with $\alpha=1 / 8$ corresponding to the limit of infinite on-site repulsion (Voit 1993). By choosing the positive direction to be from left to right, below we consider the one-dimensional vectors $\mathbf{k}$ and $\mathbf{q}$ as scalars and $k_{F} \equiv\left|\mathbf{k}_{F}\right|$. Here the ground-state momentum-distribution function $\mathbf{n}_{r}(\mathbf{k})$, with $r= \pm$ the branch index $(r=+$ indicates the branch of the right movers and $r=-$ that of the left movers), is continuous but not differentiable at $\mathbf{k}=r k_{F}$. For the density-density correlation function $\Pi_{r r}(q)$ pertaining to the interacting system we have (DL 1974; see, e.g., Metzner and Di Castro 1993, Voit 1994) 17

$$
\Pi_{r r}(q)=\frac{1}{2 \pi} \frac{g_{r}(\mathbf{q})}{i q_{0}-\mathbf{v}_{r}^{0} \cdot \mathbf{q}}, \quad g_{r}(\mathbf{q}):=\int_{-\lambda / 2+r k_{F}+\mathbf{q} / 2}^{\lambda / 2+r k_{F}-\mathbf{q} / 2} \mathrm{~d} \mathbf{k}\left[\mathrm{n}_{r}(\mathbf{k}-\mathbf{q} / 2)-\mathrm{n}_{r}(\mathbf{k}+\mathbf{q} / 2)\right],
$$

where $\mathbf{v}_{r}^{0}$ stands for the constant velocity of the non-interacting fermions; it points to the positive direction for $r=+$ and the negative direction for $r=-$. Further, $\lambda$ is a cut-off which is finite but can be arbitrarily large; for our following considerations it is required that $\lambda$ be sufficiently large so that $\mathrm{n}_{r}(\mathbf{k})$ can be replaced by zero (unity) for $r=+(r=-)$ when $\mathbf{k} \approx \lambda / 2$. The freedom to chose $\lambda$ arbitrarily large is a specific aspect of the one-dimensional Luttinger model. We note that although introduction of such a cut-off is necessary for a correct treatment of the Luttinger model (Mattis and Lieb 1965), imposition of a 'sharp' cut-off on $\mathbf{n}_{r}(\mathbf{k})$, implying $\mathbf{n}_{ \pm}\left(\mp \lambda / 2 \mp 0^{+}\right)=0$ and $\mathrm{n}_{ \pm}\left(\mp \lambda / 2 \pm 0^{+}\right)=1$, gives rise to an incorrect asymptotic result for the long-distance behaviour of in particular the single-particle Green functions $G_{r}$ pertaining to the model under consideration; ${ }^{18}$ for this long-distance asymptotic behaviour to be described correctly, it is necessary that $\mathrm{n}_{+}(-\mathbf{k})$ and $\mathrm{n}_{-}(\mathbf{k})$ are reduced to zero for $\mathbf{k} \gg \lambda / 2$, which can be achieved through imposing a 'soft' cut-off on these functions which becomes operative for $|\mathbf{k}|$ larger than $\lambda / 2$ (Farid 1999b).

Through decomposing the integral in Eq. (54) into two integrals involving separately $\mathrm{n}_{r}(\mathbf{k}-\mathbf{q} / 2)$ and $\mathrm{n}_{r}(\mathbf{k}+\mathbf{q} / 2)$ and subsequently transforming the integration variable $\mathbf{k}$, with $\mathbf{k} \rightarrow \mathbf{k}+\mathbf{q} / 2$ in the first and $\mathbf{k} \rightarrow \mathbf{k}-\mathbf{q} / 2$ in the second integral, cancellation of a common contribution to the resulting integrals gives rise to $g_{r}(\mathbf{q})=\int_{-\lambda / 2+r k_{F}}^{-\lambda / 2+\mathbf{q}} \mathrm{d} \mathbf{k} \mathbf{n}_{r}(\mathbf{k})-$ $\int_{\lambda / 2+r k_{F}-\mathbf{q}}^{\lambda / 2+r k_{F}} \mathrm{~d} \mathbf{k} \mathrm{n}_{r}(\mathbf{k})$. By assuming $\lambda$ to be sufficiently large (see above), $\mathrm{n}_{r}(\mathbf{k})$ in the first integral can be replaced by unity (zero) and in the second integral by zero (unity) for $r=+(r=-)$, upon which one readily obtains

\footnotetext{
17 The boundaries of the integral $g_{r}(\mathbf{q})$ are determined by the observation that $\mathbf{k}$ in $\mathbf{n}_{r}(\mathbf{k})$ is restricted to the range $-\lambda / 2+r k_{F} \leq$ $\mathbf{k} \leq \lambda / 2+r k_{F}$.

${ }^{18}$ By imposing a 'sharp' cut-off on $\mathbf{n}_{r}(\mathbf{k})$, the long-distance behaviour of $G_{r}$ coincides with that specific to FLs (Farid 1999b); such a behaviour is incorrect as long as $\alpha \neq 0$.
} 


$$
g_{r}(\mathbf{q})=r \mathbf{q}
$$

From this and Eq. (54) it follows that

$$
\Pi_{r r}(q)=\frac{1}{2 \pi} \frac{r \mathbf{q}}{i q_{0}-\mathbf{v}_{r}^{0} \cdot \mathbf{q}} \equiv \Pi_{r r}^{0}(q),
$$

where $\Pi_{r r}^{0}(q)$ stands for the density-density correlation function pertaining to the non-interacting system. This result is a direct manifestation of the CLT (DL 1974, Bohr 1981) which applies to the one-dimensional Luttinger model. Note that validity of Eq. (55) does not depend on any other aspect of $\mathbf{n}_{r}(\mathbf{k})$ than it be zero (unity) for $r=+(r=-)$ when $\mathbf{k}$ is sufficiently far to the right of $r k_{F}$ and unity (zero) for $r=+(r=-)$ when $\mathbf{k}$ is sufficiently far to the left of $r k_{F}$. Thus Eq. (55) holds also for the case where $\mathrm{n}_{r}(\mathbf{k})$ in Eq. (54) is replaced by its non-interacting counter-part $\Theta\left(k_{F}-r \mathbf{k}\right)$; using this step function, the validity of this statement is trivially verified by explicit calculation.

Let us now assume $|\mathbf{q}|$ to be small. Using (inappropriately) $\mathbf{n}_{r}(\mathbf{k}-\mathbf{q} / 2)-\mathrm{n}_{r}(\mathbf{k}+\mathbf{q} / 2)=-\mathbf{q} \partial \mathrm{n}_{r}(\mathbf{k}) / \partial \mathbf{k}+\mathcal{O}\left(|\mathbf{q}|^{3}\right)$ for all $\mathbf{k}$ (c.f. Eq. (16) above and Eq. (63) below), through integration-by-parts and making use of $\mathbf{n}_{r}\left(\lambda / 2+r k_{F}-\mathbf{q} / 2\right)=0(1)$ and $\mathrm{n}_{r}\left(-\lambda / 2+r k_{F}+\mathbf{q} / 2\right)=1$ (0) for $r=+(r=-)$, up to $\mathcal{O}\left(|\mathbf{q}|^{3}\right)$ we evidently obtain the (exact) result in Eq. (55), in spite of the fact that $\mathbf{n}_{r}(\mathbf{k})$ is not a differentiable function of $\mathbf{k}$ in a neighbourhood of $\mathbf{k}=r k_{F}$.

We shall now demonstrate that $\mathcal{O}\left(|\mathbf{q}|^{3}\right)$ in $g_{r}(\mathbf{q})=r \mathbf{q}+\mathcal{O}\left(|\mathbf{q}|^{3}\right)$ is incorrect. The remainder in fact is of the form $\mathcal{O}\left(|\mathbf{q}|^{1+\alpha}\right)$. Further, as the non-analytic form of this remainder suggests, the second- and higher-order expansions of $\mathrm{n}_{r}(\mathbf{k}-\mathbf{q} / 2)-\mathrm{n}_{r}(\mathbf{k}+\mathbf{q} / 2)$ (in powers of $\left.\mathbf{q}\right)$ result in divergent integrals, reflecting the fact that $1<1+\alpha<2$.

Below we consider $\int_{-\lambda_{0} / 2+r k_{F}+\mathbf{q} / 2}^{\lambda_{0} / 2+r k_{F}-\mathbf{q} / 2} \mathrm{~d} \mathbf{k}\left[\mathrm{n}_{r}(\mathbf{k}-\mathbf{q} / 2)-\mathrm{n}_{r}(\mathbf{k}+\mathbf{q} / 2)\right] \equiv \int_{-\lambda_{0} / 2+\mathbf{q} / 2}^{\lambda_{0} / 2-\mathbf{q} / 2} \mathrm{~d} \mathbf{k}\left[\mathrm{n}_{r}\left(\mathbf{k}+r k_{F}-\mathbf{q} / 2\right)-\mathrm{n}_{r}(\mathbf{k}+\right.$ $\left.\left.r k_{F}+\mathbf{q} / 2\right)\right]$ in terms of the following three constituent parts

$$
\begin{aligned}
& \mathcal{I}_{1}:=\int_{-\lambda_{0} / 2+\mathbf{q} / 2}^{-\mathbf{q} / 2} \mathrm{~d} \mathbf{k}\left[\mathrm{n}_{r}\left(\mathbf{k}+r k_{F}-\mathbf{q} / 2\right)-\mathrm{n}_{r}\left(\mathbf{k}+r k_{F}+\mathbf{q} / 2\right)\right], \\
& \mathcal{I}_{2}:=\int_{-\mathbf{q} / 2}^{\mathbf{q} / 2} \mathrm{~d} \mathbf{k}\left[\mathrm{n}_{r}\left(\mathbf{k}+r k_{F}-\mathbf{q} / 2\right)-\mathrm{n}_{r}\left(\mathbf{k}+r k_{F}+\mathbf{q} / 2\right)\right], \\
& \mathcal{I}_{3}:=\int_{\mathbf{q} / 2}^{\lambda_{0} / 2-\mathbf{q} / 2} \mathrm{~d} \mathbf{k}\left[\mathrm{n}_{r}\left(\mathbf{k}+r k_{F}-\mathbf{q} / 2\right)-\mathrm{n}_{r}\left(\mathbf{k}+r k_{F}+\mathbf{q} / 2\right)\right],
\end{aligned}
$$

where $\lambda_{0}$ is a constant which we chose to satisfy $|\mathbf{q}|<\lambda_{0} \ll \lambda$. Our choice of the inequality $\lambda_{0} \ll \lambda$ is motivated by the fact that it justifies our use of the following two-term asymptotic expression for $\mathrm{n}_{r}(\mathbf{k})$ pertaining to the one-dimensional Luttinger model for spin-less fermions (Voit 1994)

$$
\mathrm{n}_{r}\left(\mathbf{k}+r k_{F}\right) \sim \frac{1}{2}-r C \operatorname{sgn}(\mathbf{k})|\mathbf{k}|^{\alpha},
$$

where $C$ is a positive constant. Making use of the expression in Eq. (60), through exact evaluation of the integrals one obtains (below we assume $\mathbf{q}>0$ ):

$$
\begin{aligned}
\mathcal{I}_{1}=\mathcal{I}_{3} & \sim \begin{cases}\frac{r C}{\alpha+1}\left(\left(\lambda_{0} / 2\right)^{\alpha+1}-\left(\lambda_{0} / 2-\mathbf{q}\right)^{\alpha+1}-\mathbf{q}^{\alpha+1}\right), & \text { (No expansion) } \\
r C\left(\left(\lambda_{0} / 2-\mathbf{q} / 2\right)^{\alpha}-(\mathbf{q} / 2)^{\alpha}\right) \mathbf{q}, & \text { (Expansion) }\end{cases} \\
\mathcal{I}_{2} & \sim\left\{\begin{array}{lc}
\frac{2 r C}{\alpha+1} \mathbf{q}^{\alpha+1}, & \text { (No expansion) } \\
\frac{2^{1-\alpha}(\alpha+2) r C}{\alpha+1} \mathbf{q}^{\alpha+1} . & \text { (Expansion) }
\end{array}\right.
\end{aligned}
$$

The dissimilarity of the results based upon the first-order expansion of $\mathbf{n}_{r}\left(\mathbf{k}+r k_{F}-\mathbf{q} / 2\right)-\mathrm{n}_{r}\left(\mathbf{k}+r k_{F}+\mathbf{q} / 2\right)$, indicated by "Expansion", from the exact results, indicated by "No expansion", confirms our above statement concerning the invalidity of the expansion procedure. Note for instance that $\mathcal{I}_{2}$ as determined through expansion coincides with the exact result only when $\alpha=2$. It is interesting also to note that contrary to the expression $\mathbf{n}_{r}(\mathbf{k}-\mathbf{q} / 2)-\mathrm{n}_{r}(\mathbf{k}+\mathbf{q} / 2)=$ $-\mathbf{q} \partial \mathbf{n}_{r}(\mathbf{k}) / \partial \mathbf{k}+\mathcal{O}\left(|\mathbf{q}|^{3}\right)$ which implies absence of a zeroth-order term in $\mathbf{q}$, from Eq. (60) one in fact has (c.f. Eq. (16) above)

$$
\begin{array}{r}
\mathrm{n}_{r}(\mathbf{k}-\mathbf{q} / 2)-\mathrm{n}_{r}(\mathbf{k}+\mathbf{q} / 2) \approx 2 r C \operatorname{sgn}(\mathbf{q})\left|\mathbf{k}-r k_{F}\right|^{\alpha}+\alpha r C\left|\mathbf{k}-r k_{F}\right|^{\alpha-1} \mathbf{q} \\
\text { when } \mathbf{k} \in\left[r k_{F}-\mathbf{q} / 2, r k_{F}+\mathbf{q} / 2\right]
\end{array}
$$


which contains a zeroth-order term in $\mathbf{q}$ as long as $\mathbf{k} \neq r k_{F}$. Without taking into account the zeroth-order term in Eq. (63), we would have $\mathcal{I}_{2} \sim 2^{1-\alpha} r C \mathbf{q}^{\alpha+1}$ (Expansion) rather than the '(Expansion)' result for $\mathcal{I}_{2}$ in Eq. (62); the two results coincide only for $\alpha=1$.

Adding the three contributions $\mathcal{I}_{j}, j=1,2,3$, as presented in Eqs. 61) and (62), we obtain

$$
\begin{array}{ll}
\int_{-\lambda_{0} / 2+r k_{F}+\mathbf{q} / 2}^{\lambda_{0} / 2+r k_{F}-\mathbf{q} / 2} \mathrm{~d} \mathbf{k}\left[\mathrm{n}_{r}(\mathbf{k}-\mathbf{q} / 2)-\mathrm{n}_{r}(\mathbf{k}+\mathbf{q} / 2)\right] & \text { (No expansion) } \\
\sim \begin{cases}\frac{2 r C}{\alpha+1}\left(\left(\lambda_{0} / 2\right)^{\alpha+1}-\left(\lambda_{0} / 2-\mathbf{q}\right)^{\alpha+1}\right), & \text { (Expansion) } \\
\frac{2^{1-\alpha}(\alpha+2) r C}{\alpha+1} \mathbf{q}^{\alpha+1}+2 r C\left(\left(\lambda_{0} / 2-\mathbf{q} / 2\right)^{\alpha}-(\mathbf{q} / 2)^{\alpha}\right) . & \end{cases}
\end{array}
$$

Assuming $0<\mathbf{q} \ll \lambda_{0}$, and neglecting $\mathbf{q}^{\alpha+1}$, both of the results in Eq. (64) reduce to $2^{1-\alpha} r C \lambda_{0}^{\alpha} \mathbf{q}$. This analysis shows that the error in $g_{r}(\mathbf{q})$ as obtained through the first-order expansion of the integrand of $g_{r}(\mathbf{q})$ is of order $|\mathbf{q}|^{\alpha+1}$ and not of order $|\mathbf{q}|^{3}$. As we have indicated above, in practice $\alpha$ is small (recall that for the one-dimensional Hubbard model, $0<\alpha<1 / 8)$, so that the range of the $\mathbf{q}$ values for which $|\mathbf{q}|$ is dominant with respect to $|\mathbf{q}|^{\alpha+1}$ is substantially reduced as compared with the range for which $|\mathbf{q}|$ is dominant with respect to $|\mathbf{q}|^{3}$. Note in passing that $|\mathbf{q}|^{\alpha+1}=|\mathbf{q}|^{3}$ provided $\alpha=2$ which coincides with the condition required for the equality of the $\mathcal{I}_{2}$ 's as evaluated without relying upon expansion and through (correct) expansion of the integrand of $\mathcal{I}_{2}$.

In $d>1$, contrary to $d=1$ (in particular when dealing with the one-dimensional Luttinger model), the radial cut-off parameter $\lambda$ cannot be arbitrarily large. Consequently, the condition $|\mathbf{q}| \ll \lambda_{0}$ is extremely restrictive, owing to $\lambda_{0} \ll \lambda \ll\left\|\mathbf{k}_{F}\right\|$, and therefore should be relaxed. Correspondingly, evaluation of integrals subsequent to an expansion of the pertinent integrands yields results which even in the leading order deviate from the exact results (see Eqs. (61), (62) and (64) above).

Finally, the momentum distribution function in Eq. (60) is peculiar in that $\mathrm{n}_{r}(\mathbf{k})$ does not allow for a Taylor expansion in a neighbourhood of $\mathbf{k}=r k_{F}$. In the case of the conventional FLs, although $\mathrm{n}(\mathbf{k})$ is discontinuous at $\mathbf{k}=\mathbf{k}_{F}$, nevertheless it possesses regular (Taylor) expansions to the left and to the right of $\mathbf{k}=\mathbf{k}_{F}$. 19 Consequently, in cases where one has to do with conventional FLs, provided one takes appropriate care of the discontinuity at $\mathbf{k}=\mathbf{k}_{F}$ of the associated $n(\mathbf{k})$, the procedure based upon expansion of $n(\mathbf{k}-\mathbf{q} / 2)-n(\mathbf{k}+\mathbf{q} / 2)$ yields correct results to the expected order. One observes that mathematical procedures that are in general inapplicable, may be applied, with due care, when dealing with conventional FLs (see Farid 1999a). The same procedure fails however, in the case of unconventional FLs or NFLs.

\section{SUMMARY AND CONCLUDING REMARKS}

In this work we have presented a brief outline of the steps that underlie a generalised method which has been modelled on a formalism due to DL (1974) which exactly solves the one-dimensional Luttinger (1963) model. Key elements in both the original DL (1974) method and its generalisation (MCDC 1998) are the conservation laws and the associated Ward identities. These identities give rise to some auxiliary relationships which are exact in the one-dimensional Luttinger model, rendering this model exactly solvable, whereas though not exact, these auxiliary relationships have been asserted to be asymptotically exact for Fermi systems in $d>1$ in the limit where the scattering of the electrons in a thin layer circumscribing the Fermi surface is strictly in the forward-scattering channel (corresponding to scattering processes with vanishingly-small momentum transfers, that is $\mathbf{q} \rightarrow \mathbf{0}$ ). We have presented an analysis concerning a crucial relationship between the irreducible density and current vertex parts, $\Lambda_{\sigma}^{0}(p ; q)$ and $\boldsymbol{\Lambda}_{\sigma}(p ; q)$ respectively, which is exactly valid in the case of the one-dimensional Luttinger model and asserted (MCDC 1998) to be asymptotically exact in the forward-scattering limit when $d>1$. We have explicitly demonstrated failure of this relationship for the conventional FL systems in $d=2$. We have further demonstrated that this relationship is exact, in any arbitrary spatial dimension, only in the trivial case of systems of strictly non-interacting fermions.

\footnotetext{
${ }^{19}$ Within the framework of the RPA, $\mathrm{n}(\mathbf{k})$ pertaining to the uniform system of electrons (in $d=3$ ) has been shown to possess finite left and right derivatives at $\mathbf{k}=\mathbf{k}_{F}$ (Daniel and Vosko 1960). On the other hand, for a uniform system of electrons interacting through a hard-core two-body potential, Belyakov (1961) (see also Sartor and Mahaux 1980) has obtained an $\mathrm{n}(\mathbf{k})$ which has logarithmically divergent derivatives to the left and right of $\mathbf{k}=\mathbf{k}_{F}$. For a discussion of the behaviour of $\mathbf{n}(\mathbf{k})$ in the close vicinity of $\mathbf{k}=\mathbf{k}_{F}$ see (Farid 1999a).
} 
20 From the same analysis it follows that for NFLs in $d>1$ there does not exist even the remotest ground on the basis of which the presumed asymptotic validity of the mentioned relationship can be justified: here, the interlocking of energies and momenta in the arguments of the corresponding self-energies, which by the very fact of being by definition non-differentiable in neighbourhoods of the Fermi energy and/or the Fermi momentum (otherwise the associated metallic states would not be NFLs), stands in the way of obtaining a relationship similar in appearance to the indicated supposed asymptotic relationship between the irreducible vertex parts.

We have further explicitly demonstrated that the continuity equation only in $d=1$ gives rise to a direct relationship between the vertex parts $\Lambda_{\sigma}^{\nu}(p ; q)$ and $\Gamma_{\sigma}^{\nu}(p ; q), \nu=0,1, \ldots, d$, independent of the analytic properties of the momentum distribution function $\mathrm{n}_{\sigma}(\mathbf{k})$. In $d>1$, continuity as well as continuous differentiability of this function in the aboveindicated narrow band of the momentum space circumscribing the Fermi surface must be assumed before a similar direct relationship between the two vertex parts, as in $d=1$, can be deduced. As is well known, $\mathrm{n}_{\sigma}(\mathbf{k})$ is discontinuous at $\mathbf{k}=\mathbf{k}_{F}$ both for FLs and certain class of NFLs (for the latter see Farid 1999a). For the case of the one-dimensional Luttinger model, though continuous, $\mathrm{n}_{\sigma}(\mathbf{k})$ is not differentiable at $\mathbf{k}=\mathbf{k}_{F}$. Therefore, by assuming a similar behaviour for $\mathbf{n}_{\sigma}(\mathbf{k})$ in $d>1$ as in $d=1$, we have established that the indicated simplified relationships between $\Lambda_{\sigma}^{\nu}(p ; q)$ and $\Gamma_{\sigma}^{\nu}(p ; q), \nu=0,1, \ldots, d$, do not hold in $d>1$.

Each of the above-mentioned two findings removes ground for validity in $d>1$ of the heretofore supposed asymptotic Ward identities in the forward-scattering limit. This failure concerns both FLs (excluding strictly non-interacting metallic systems) and NFLs.

In an earlier work (Farid 1999b) we have demonstrated failure of the Haldane (1992) bosonisation scheme in $d>1$ both on general grounds and on grounds of an explicit calculation on a model of interacting fermions in $d=2$. Within the framework of the Haldane bosonisation scheme, the explicit expression for the single-particle Green function $G$ takes a simple form in the space-time representation. It is interesting that through replacing a screening function that features in this expression by its random-phase approximation (that is RPA) counterpart, one obtains an expression which coincides with that obtained within the generalised DL framework; compare Eq. (5.65) in (MCDC 1998) with for instance Eq. (24) in (Kwon, Houghton and Marston 1995). The use of the RPA within the framework of the generalised DL method is considered to be justified through reliance upon the so-called generalised CLT (Kopietz, Hermisson and Schönhammer 1996) which implies that while the RPA yields the exact response function in the case of the one-dimensional Luttinger model (DL 1974) (see Eq. (56) above), it yields an asymptotically exact response function in $d>1$ in the forward-scattering limit. In (Farid 1999b) we have commented on the generalised CLT, however independent of the true status of this theorem in $d>1$, and whether one has to do with FL or NFL systems, from the point of view of our considerations in the present work the coincidence of the two Green functions is of crucial importance as it indirectly establishes that indeed the generalised DL (MCDC 1998) formalism is of no validity for systems of interacting fermions in $d>1$.

To summarise the main conclusions arrived at in this and our earlier works (Farid 1999a,b), we can state that none of the presumed non-perturbative methods designed thus far for investigating the existence or otherwise of NFL metallic states in $d>1$ has proved capable of dealing with systems of interacting fermion in $d>1$, not even in the weakly-interacting limit. Renewed attempts concerning design and application of non-perturbative methods are therefore called for in order to settle the long-standing problem with regard to the existence or otherwise of NFL metallic states in spatial dimensions greater than one. It is therefore befitting to close this work by mentioning that Laughlin's (1998) recent statement, that "... and all attempts to account for the existence of a Luttinger-liquid at zero temperature in spatial dimension greater than one have failed.", must needs be qualified and in due course perhaps revoked altogether.

\section{ACKNOWLEDGEMENTS}

It is a pleasure for me to thank Professor Peter B. Littlewood and members of the Theory of Condensed Matter Group for their kind hospitality at Cavendish Laboratory where this work was completed. I record my indebtedness to Girton College, Cambridge, for invaluable support. I extend my thanks to Dr Peter Kopietz for kindly providing

\footnotetext{
${ }^{20}$ Recall that metallic states of non-interacting fermions are ideal FLs.
} 
me with references (Kopietz 1997) and (Bohr 1981). I dedicate this work to the memory of Lady Bertha Jeffreys (1903 - 1999).

\section{APPENDIX A: ON DIMENSIONAL CONTINUATION AND ITS APPLICATION TO INTERACTING FERMI SYSTEMS}

In this Appendix we first present a brief account of the technique of dimensional continuation (Wilson 1973, appendix). 21 Subsequently, we point out that the formalism of interacting fermions even in the limit of strong forward scattering cannot be subjected to the conventional process of dimensional continuation. We consequently propose an unconventional approach to this process which involves one continuous free parameter and therefore defines an infinite family of continuations that correctly interpolate between $d=1$ and $d=2$; they also correctly interpolate between $d=2$ and $d=3$ for the class of cylindrically symmetric integrands.

Consider

$$
\mathcal{J}_{d}(\mathbf{q}):=\int_{S} \mathrm{~d}^{d} \mathbf{k} f_{\mathbf{q}}(\mathbf{k})
$$

where $d$ is some real or complex number. Let $\left\{\mathbf{e}_{1}, \ldots, \mathbf{e}_{J}\right\}$ be an orthonormal basis spanning the so-called parallel space (to whose complimentary space we refer as tangent space), large enough to accommodate the vector $\mathbf{q}$ (in general, all external momenta $\left\{\mathbf{q}_{j}\right\}$ upon which the integrand may depend). We have

$$
S \equiv S_{\|} \cup S_{\perp}
$$

With

$$
\mathbf{k}=\mathbf{k}_{\|}+\mathbf{k}_{\perp} \equiv \sum_{j=1}^{J} k_{\|}^{j} \mathbf{e}_{j}+\mathbf{k}_{\perp}
$$

one can therefore write

$$
\mathcal{J}_{d}(\mathbf{q})=\int_{S_{\|}} \mathrm{d} k_{\|}^{1} \ldots \mathrm{d} k_{\|}^{J} \int_{S_{\perp}} \mathrm{d}^{d-J} \mathbf{k}_{\perp} f_{\mathbf{q}}(\mathbf{k}) .
$$

By assuming independence of $f_{\mathbf{q}}(\mathbf{k})$ upon direction of $\mathbf{k}_{\perp}$, i.e. by assuming

$$
f_{\mathbf{q}}(\mathbf{k}) \equiv \tilde{f}_{\mathbf{q}}(\mathbf{k} \cdot \mathbf{k}, \mathbf{k} \cdot \mathbf{q})
$$

one has

$$
\int_{S_{\perp}} \mathrm{d}^{d-J} \mathbf{k}_{\perp} f_{\mathbf{q}}(\mathbf{k})=K_{d-J} \int_{0}^{\infty} \mathrm{d}\left\|\mathbf{k}_{\perp}\right\|\left\|\mathbf{k}_{\perp}\right\|^{d-J-1} \tilde{f}_{\mathbf{q}}(\mathbf{k} \cdot \mathbf{k}, \mathbf{k} \cdot \mathbf{q}),
$$

where

$$
K_{\nu}:=\frac{2 \pi^{\nu / 2}}{\Gamma(\nu / 2)},
$$

with $\Gamma(z)$ the gamma function. The value of $K_{\nu}$ in Eq. (A7) is equal to the area of the hyper-sphere of unit radius in $\nu$ dimensions. The $J+1$ integrals over $k_{\|}^{1}, \ldots, k_{\|}^{J}$ and $\left\|\mathbf{k}_{\perp}\right\|$ are conventional and are evaluated in the usual way. It should be evident that the key element for reducing the abstract integral in Eq. (A1) into a conventional multidimensional integral is the assumption specified by the expression in Eq. (A5). Below we explicitly consider two cases which clarify the role played by the dimension of the parallel space, $J$.

Case I: Let $J=1$. Let further $\theta$ denote the planar angle between $\mathbf{k}$ and $\mathbf{q}$. With

\footnotetext{
${ }^{21}$ For a comprehensive review of this topic see Collins (1984, Chapter 4).
} 


$$
\left\|\mathbf{k}_{\perp}\right\|=\|\mathbf{k}\| \sin \theta, \quad k_{\|}^{1}=\|\mathbf{k}\| \cos \theta, \quad \theta \in[0, \pi]
$$

one has $\mathrm{d} k_{\|}^{1} \mathrm{~d}\left\|\mathbf{k}_{\perp}\right\|=\mathrm{d} \theta \mathrm{d}\|\mathbf{k}\|\|\mathbf{k}\|,\left\|\mathbf{k}_{\perp}\right\|^{d-2}=\|\mathbf{k}\|^{d-2}(\sin \theta)^{d-2}$ and thus (Wilson 1973)

$$
\begin{aligned}
\mathcal{J}_{d}(\mathbf{q}) & =\int_{-\infty}^{\infty} \mathrm{d} k_{\|}^{1} K_{d-1} \int_{0}^{\infty} \mathrm{d}\left\|\mathbf{k}_{\perp}\right\|\left\|\mathbf{k}_{\perp}\right\|^{d-2} \tilde{f}_{\mathbf{q}}(\mathbf{k} \cdot \mathbf{k}, \mathbf{k} \cdot \mathbf{q}) \\
& \equiv K_{d-1} \int_{0}^{\pi} \mathrm{d} \theta \int_{0}^{\infty} \mathrm{d}\|\mathbf{k}\|\|\mathbf{k}\|^{d-1}(\sin \theta)^{d-2} \tilde{f}_{\mathbf{q}}\left(\|\mathbf{k}\|^{2},\|\mathbf{k}\|\|\mathbf{q}\| \cos \theta\right) .
\end{aligned}
$$

Since $K_{d-1}(\sin \theta)^{d-2} \rightarrow \delta(\theta)+\delta(\theta-\pi)$ for $d \downarrow 1$, it follows that in the limit $d \downarrow 1$ the expression on the RHS of Eq. (A9) recovers the pertinent expression for $d=1$, i.e.

$$
\begin{aligned}
\left.\mathcal{J}_{d}(\mathbf{q})\right|_{d \downarrow 1} & =\int_{0}^{\infty} \mathrm{d}\|\mathbf{k}\| \tilde{f}_{\mathbf{q}}\left(\|\mathbf{k}\|^{2},\|\mathbf{k}\|\|\mathbf{q}\|\right)+\int_{0}^{\infty} \mathrm{d}\|\mathbf{k}\| \tilde{f}_{\mathbf{q}}\left(\|\mathbf{k}\|^{2},-\|\mathbf{k}\|\|\mathbf{q}\|\right) \\
& \equiv \int_{-\infty}^{\infty} \mathrm{d} k \tilde{f}_{\mathbf{q}}\left(k^{2}, k\|\mathbf{q}\|\right) .
\end{aligned}
$$

For $d=2$, for which $K_{d-1}=2$ holds, we have

$$
\begin{aligned}
\left.\mathcal{J}_{d}(\mathbf{q})\right|_{d=2} & =2 \int_{0}^{\pi} \mathrm{d} \theta \int_{0}^{\infty} \mathrm{d}\|\mathbf{k}\|\|\mathbf{k}\| \tilde{f}_{\mathbf{q}}\left(\|\mathbf{k}\|^{2},\|\mathbf{k}\|\|\mathbf{q}\| \cos \theta\right) \\
& \equiv \int_{0}^{2 \pi} \mathrm{d} \varphi \int_{0}^{\infty} \mathrm{d}\|\mathbf{k}\|\|\mathbf{k}\| \tilde{f}_{\mathbf{q}}\left(\|\mathbf{k}\|^{2},\|\mathbf{k}\|\|\mathbf{q}\| \cos \varphi\right) .
\end{aligned}
$$

The latter result, whose validity can be readily verified, ${ }^{22}$ makes explicit that for $d=2$ one indeed recovers the conventional integral over $\mathbf{k}$ of $\tilde{f}_{\mathbf{q}}\left(\|\mathbf{k}\|^{2}, \mathbf{k} \cdot \mathbf{q}\right)$ in terms of the cylindrical-polar coordinates $(\|\mathbf{k}\|, \varphi)$, with $\mathbf{q}$ the polar axis. It should be noted however, that not all two-dimensional integrals can be viewed as the $d \uparrow 2$ limit of the expression on the RHS of Eq. (A9).

Case II: Let $J=2$, so that $\left\{\mathbf{e}_{1}, \mathbf{e}_{2}\right\}$ spans the parallel space. With $\varphi$ denoting the planar angle between $\mathbf{k}_{\|}$and $\mathbf{e}_{1}$ (which without loss of generality we assume to be along $\mathbf{q}$ ) and $\theta$ that between $\mathbf{k}$ and $\mathbf{k}_{\perp}$ (i.e. $\mathbf{k}_{\perp}$ is taken as the polar axis), one has

$$
\left\|\mathbf{k}_{\perp}\right\|=\|\mathbf{k}\| \cos \theta, \quad k_{\|}^{1}=\|\mathbf{k}\| \sin \theta \cos \varphi, \quad k_{\|}^{2}=\|\mathbf{k}\| \sin \theta \sin \varphi, \quad \theta \in[0, \pi / 2], \quad \varphi \in[0,2 \pi],
$$

so that $\mathrm{d} k_{\|}^{1} \mathrm{~d} k_{\|}^{2} \mathrm{~d}\left\|\mathbf{k}_{\perp}\right\|=\mathrm{d} \varphi \mathrm{d} \theta \mathrm{d}\|\mathbf{k}\|\|\mathbf{k}\|^{2} \sin \theta,\left\|\mathbf{k}_{\perp}\right\|^{d-3}=\|\mathbf{k}\|^{d-3}(\cos \theta)^{d-3}$ and thus

$$
\begin{aligned}
\mathcal{J}_{d}(\mathbf{q})= & \int_{-\infty}^{\infty} \mathrm{d} k_{\|}^{1} \mathrm{~d} k_{\|}^{2} K_{d-2} \int_{0}^{\infty} \mathrm{d}\left\|\mathbf{k}_{\perp}\right\|\left\|\mathbf{k}_{\perp}\right\|^{d-3} \tilde{f}_{\mathbf{q}}(\mathbf{k} \cdot \mathbf{k}, \mathbf{k} \cdot \mathbf{q}) \\
= & K_{d-2} \int_{0}^{2 \pi} \mathrm{d} \varphi \int_{0}^{\pi / 2} \mathrm{~d} \theta(\cos \theta)^{d-3} \sin \theta \\
& \times \int_{0}^{\infty} \mathrm{d}\|\mathbf{k}\|\|\mathbf{k}\|^{d-1} \tilde{f}_{\mathbf{q}}\left(\|\mathbf{k}\|^{2},\|\mathbf{k}\|\|\mathbf{q}\| \sin \theta \cos \varphi\right) .
\end{aligned}
$$

Since $K_{d-2}(\cos \theta)^{d-3} \rightarrow \delta(\theta-\pi / 2)$ for $d \downarrow 2$, it follows that

$$
\left.\mathcal{J}_{d}(\mathbf{q})\right|_{d \downarrow 2}=\int_{0}^{2 \pi} \mathrm{d} \varphi \int_{0}^{\infty} \mathrm{d}\|\mathbf{k}\|\|\mathbf{k}\| \tilde{f}_{\mathbf{q}}\left(\|\mathbf{k}\|^{2},\|\mathbf{k}\|\|\mathbf{q}\| \cos \varphi\right)
$$

\footnotetext{
${ }^{22}$ To this end employ the variable transformation $\varphi \rightarrow u$ defined through $u=\cos \varphi$, implying $\mathrm{d} \varphi=\mp \mathrm{d} u /\left(1-u^{2}\right)^{1 / 2}$ for $\varphi \in[0, \pi]$ and $\varphi \in[\pi, 2 \pi]$ respectively, and a subsequent reverse transformation $u \rightarrow \varphi$.
} 
which is indeed the expected expression for a two-dimensional integral in the cylindrical-polar coordinates $(\|\mathbf{k}\|, \varphi)$. However, again as in the case considered above (concerning $J=1$ ), not all two-dimensional integrals over arbitrary functions of $\mathbf{k}$ can be obtained as the $d \downarrow 2$ limit of the $d$-dimensional integral in Eq. (A13).

The above considerations make explicit that whereas by an appropriate choice of the dimension of the parallel space one can analytically continue $d$ and obtain the standard integrals for integer dimensions, nonetheless the applicability of this procedure in an essential way depends on the requirement that $f_{\mathbf{q}}(\mathbf{k})$ in so far as its dependence upon $\mathbf{k}$ is concerned, be a function of at most $\mathbf{k} \cdot \mathbf{k}$ and $\mathbf{k} \cdot \mathbf{q}$. In cases where the dependence of $f_{\mathbf{q}}(\mathbf{k})$ upon $\mathbf{k}$ is more general, the conventional process of dimensional continuation may be possible in the limit of small $\|\mathbf{q}\|$. In this limit, provided $f_{\mathbf{q}}(\mathbf{k})$ be continuously differentiable in a neighbourhood of $\mathbf{q}=\mathbf{0}$, it may be possible (that is, under the condition that $f_{\mathbf{q}}(\mathbf{k})$ to linear order in $\mathbf{q}$ can be described by a function of $\mathbf{k} \cdot \mathbf{k}$ and $\mathbf{k} \cdot \mathbf{q}$ ) to carry through the process of dimensional continuation, accurate to linear order in $\mathbf{q}$. 23 This is precisely the case in the generalised DL formalism by MCDC (1998) (see the last part of $\S$ II).

It is readily verified that the following expression, whose origin does not lie in the conventional formalism for dimensional continuation (Wilson 1973), is a smooth analytic continuation for $d \in(1,2]$ :

$$
\begin{aligned}
\mathcal{J}_{d}(\mathbf{q}):= & \frac{K_{d-1}}{\vartheta(d)}\left\{\int_{0}^{\pi} \mathrm{d} \theta(\sin \theta)^{d-2} \int_{0}^{\infty} \mathrm{d}\|\mathbf{k}\|\|\mathbf{k}\|^{d-1} \bar{f}_{\mathbf{q}}(\|\mathbf{k}\|, \theta)\right. \\
& \left.+\int_{\pi}^{\vartheta(d) \pi} \mathrm{d} \theta(\sin (\theta-\pi))^{d-2} \int_{0}^{\infty} \mathrm{d}\|\mathbf{k}\|\|\mathbf{k}\|^{d-1} \bar{f}_{\mathbf{q}}(\|\mathbf{k}\|, \theta)\right\},
\end{aligned}
$$

where

$$
\bar{f}_{\mathbf{q}}(\|\mathbf{k}\|, \theta) \equiv f_{\mathbf{q}}(\mathbf{k}),
$$

with $(\|\mathbf{k}\|, \theta)$ the cylindrical-polar coordinates of $\mathbf{k}$, and (see Figure)

$$
\vartheta(d):=1+(3-d) \exp \left(a\left[1-1 /(d-1)^{1 / a}\right]\right), \quad \text { with } \quad a>0 .
$$

We note that the integral in Eq. (A15) together with $\vartheta(d)$ in Eq. (A17) is also correct for $d=3$ over the space of functions with cylindrical symmetry; therefore it is also a smooth continuation for $d \in[2,3]$, however over a restricted set of functions. In this respect Eq. (A15) is of limited validity for $d>2$, in the same way that Eq. (A9) is of limited validity for $d>1$.

The choice for $\vartheta(d)$ in Eq. (A17) is not unique. However, in choosing any $\vartheta(d)$ interpolating between $\vartheta(1)=1$ and $\vartheta(2)=2$ (and possibly $\vartheta(3)=1$, etc.) care must be exercised that in the limit $d \rightarrow 1$ the singularities of $K_{d-1}(\sin \theta)^{d-2}$ and $K_{d-1}(\sin (\theta-\pi))^{d-2}$ for respectively $\theta \uparrow \pi$ and $\theta \downarrow \pi$ do not yield repeated contributions. This is prevented by the requirement $(\vartheta(d)-1)^{d-1} \rightarrow 0$ for $d \downarrow 1$; in consequence of this, for $d \downarrow 1$ the reduction in the width of the interval $[\pi, \vartheta(d) \pi]$ renders the growth in the integrand, due to the singularity of $K_{d-1}(\sin (\theta-\pi))^{d-2}$ at $d=1$, ineffective. It can be easily verified that $\vartheta(d)$ in Eq. (A17) indeed satisfies this requirement. The freedom in choice of the continuous positive parameter $a$ in Eq. (A17) implies that the dimensional continuation scheme as prescribed by Eqs. (A15) and A17) represents an infinite family of dimensional continuations. However, in order to recover the expression in Eq. (A9), applicable to functions $\left\{f_{\mathbf{q}}(\mathbf{k})\right\}$ whose dependence upon $\mathbf{k}$ is through $\mathbf{k} \cdot \mathbf{k}$ and $\mathbf{k} \cdot \mathbf{q}$, it is required that $0<a \ll 1$ (see Figure); in the limit $a \downarrow 0$ the expressions in Eqs. (A9) and (A15) coincide.

REFERENCES

\footnotetext{
${ }^{23}$ We note that here we only deal with scalar functions $\left(f_{\mathbf{q}}(\mathbf{k})\right)$ which are functions of scalar products, however, the technique of dimensional continuation is also applicable to tensor functions. See Wilson (1973, appendix) and Collins (1984, Chapter 4).
} 
Anderson, P. W., 1988, in Frontiers and Borderlines in Many-Particle Physics, J. R. Schrieffer and R. A. Broglia (editors) (Amsterdam: North Holland), pp. 1-40;

- 1989, in Strong Correlation and Superconductivity, H. Fukuyama, S. Maekawa and A. P. Malozemoff (editors) (Berlin: Springer Verlag), pp. 2-13;

- 1990a, Phys. Rev. Lett., 64, 1839;

- 1990b, Phys. Rev. Lett., 65, 2306;

- 1991, Phys. Rev. Lett., 66, 3226;

- 1993, Phys. Rev. Lett., 71, 1220;

— 1997, THE Theory of Superconductivity in the High- $T_{c}$ Cuprate Superconductors (Princeton, NJ: Princeton University Press).

Bares, P.-A., and Wen, X.-G., 1993, Phys. Rev. B, 48, 8636.

Belyakov, V. A., 1961, Soviet Phys. - JETP, 13, 850.

Bloch, F., 1933, Z. Phys., 81, 363;

- 1934, Helv. Phys. Acta, 7, 385.

Bloom, P., 1975, Phys. Rev. B, 12, 125.

Bohr, T., 1981, The Luttinger Model Lecture notes in the series on Low-Dimensional Statistical Mechanics (Demark: NORDITA preprint, NORDITA-81/4).

Castellani, C., and Di Castro, C., 1994, Physica C, 235-240, 99.

Castellani, C., Di Castro, C., and Metzner, W., 1994, Phys. Rev. Lett., 72, 316.

Coleman, S., 1975, Phys. Rev. B, 11, 2088.

Collins, J. C., 1984, Renormalisation (Cambridge: Cambridge University Press).

Daniel, E., and Vosko, S. H., 1960, Phys. Rev., 120, 2041.

Di Castro, C., and Metzner, W., 1991, Phys. Rev. Lett., 67, 3852.

DuBois, D. F., 1959, Ann. Phys. (N.Y.), 8, 24.

Dzyaloshinskiľ, I. E. and Larkin, A. I., 1974, Soviet Phys. - JETP, 38, 202.

Engelbrecht, J. R., and Randeria, M., 1990, Phys. Rev. Lett., 65, 1032;

- 1991, Phys. Rev. Lett., 66, 3225.

Everts, H. U., and Schulz, H., 1974, Solid State Commun., 15, 1413.

FARID, B., 1999a, Phil. Mag. B 79 1097;

- 1999b, submitted.

Fujimoto, S., 1990, J. Phys. Soc. Jpn, 59, 2316.

Fukuyama, H., Narikiyo, O., and Hasegawa, Y., 1991, J. Phys. Soc. Jpn, 60, 372.

Goddard, P., and Olive, D., 1986, Int. J. Mod. Phys., 1, 303.

Haldane, F. D. M., 1980, Phys. Rev. Lett., 45, 1358;

- 1981, J. Phys. C, 14, 2585;

- 1992, Proceedings of the International School of Physics "Enrico Fermi", Course 121, J. R. Schrieffer and R. A. Broglia (editors) (New York: North Holland), pp. 5 - 30.

Hodges, C., Smith, H., and Wilkins, J. W., 1971, Phys. Rev. B, 4, 302.

Houghton, A., Kwon, H.-J., and Marston, J. B., 1994, Phys. Rev. B, 50, 1351.

Houghton, A., Kwon, H.-J., Marston, J. B., and Shankar, R., 1994, J. Phys. C, 6, 4909.

Houghton, A., and Marston, J. B., 1993, Phys. Rev. B, 48, 7790.

Jordan, P., 1935, Z. Phys., 93, 464;

- 1936a, ibid., 98, 759;

- 1936b, ibid., 99, 109.

Kopietz, P., 1997, Bosonization of Interacting Fermions in Arbitrary Dimensions in Lecture Notes in Physics. New Series m: Monographs (Berlin: Springer).

Kopietz, P., Hermisson, J., and Schönhammer, K., 1995, Phys. Rev. B, 52, 10877.

Krönig, R. de L., 1935a, Physica, 2, 491;

- 1935b, ibid., 854;

- 1935c, ibid., 968 .

Kwon, H.-J., Houghton, A., and Marston, J. B., 1995, Phys. Rev. B, 52, 8002.

Laughlin, R. B., 1998, Adv. Phys., 47, 943.

Littlewood, P. B., and Varma, C. M., 1991, J. Appl. Phys., 69, 4979.

Luther, A., 1979, Phys. Rev. B, 19, 320.

Luther, A., and Peschel, I., 1974, Phys. Rev. B, 9, 2911.

Luttinger, J. M., 1961, Phys. Rev., 121, 942;

- 1963, J. Math. Phys., 4, 1154.

Mattis, D. C., and Lieb, E. H., 1965, J. Math. Phys., 6, 304.

Metzner, W., Castellani, C., and Di Castro, C., 1998, Adv. Phys., 47, 317.

Metzner, W., and Di Castro, C., 1993, Phys. Rev. B, 47, 16107.

Sartor, R., and Mahaux, C., 1980, Phys. Rev. C, 21, 1546. 
Skyrme, T. H. R., 1958, Proc. Roy. Soc. A, 247, 260;

- 1959, ibid., 252, 236;

- 1961a, ibid., 260, 127;

- 1961b, ibid., 262, 237.

Stamp, P. C. E., 1993, J. Phys. I Paris, 3, 625.

Stone, M., 1994, Bosonization (Singapore: World Scientific).

Tomonaga, S., 1950, Prog. Theor. Phys. (Kyoto), 5, 544.

Ueda, K., and Rice, T. M., 1984, Phys. Rev. B, 29, 1514.

Varma, C. M., Littlewood, P. B., Schmitt-Rink, S., Abrahams, E., and Ruckenstein, A. E., 1989, Phys. Rev. Lett., 63, 1996;

1990 (E), Phys. Rev. Lett., 64, 497.

Voit, J., 1993, J. Phys. C, 5, 8305;

- 1994, Rep. Prog. Phys., 57, 977.

Wilson, K. G., 1973, Phys. Rev. D, 7, 2911.

Wilson, K. G., and Fisher, M. E., 1972, Phys. Rev. Lett., 28, 240.

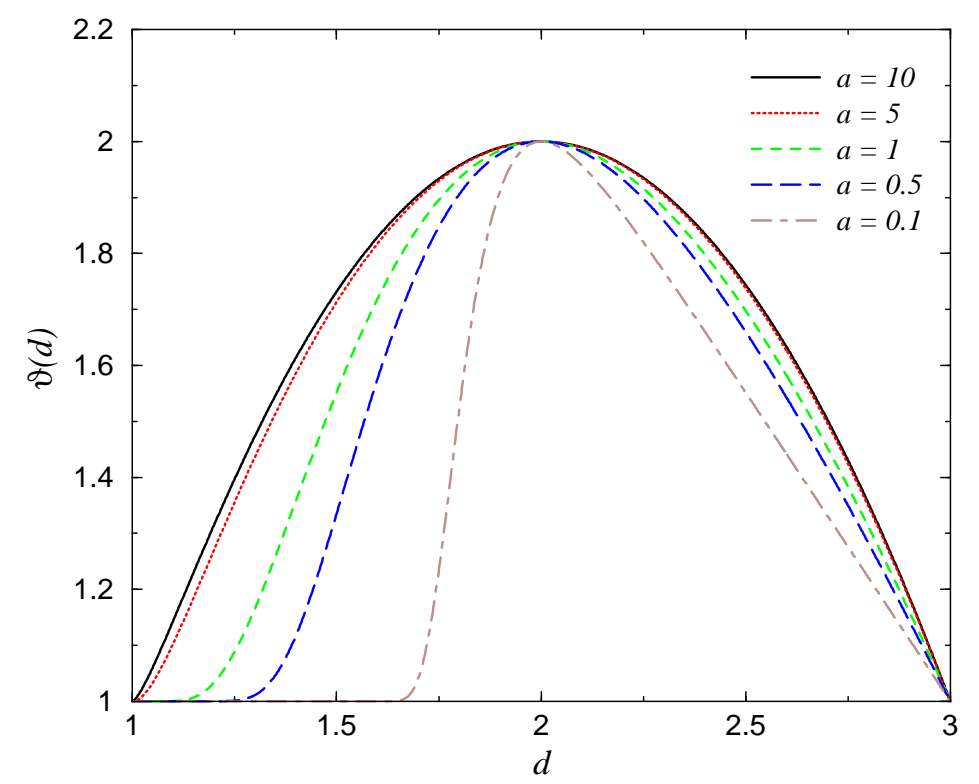

FIG. 1. The function $\vartheta(d)$ as defined in Eq. A17 for five distinct values of the continuous parameter $a$. For $a \downarrow 0$, Eq. A15) reduces to the conventional expression in Eq. (A9) for $d$-dimensional integration, with $d \in[1,2]$. 